\title{
Long-term starspot evolution, activity cycle, and orbital period variation of V711 Tauri (HR 1099) ${ }^{\star}$
}

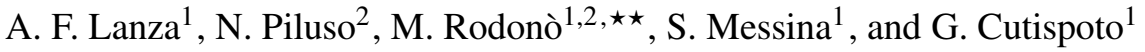 \\ 1 INAF - Osservatorio Astrofisico di Catania, via S. Sofia, 78 - 95123 Catania, Italy \\ e-mail: [nlanza;piluso; smessina; gcutispoto]@oact.inaf.it \\ 2 Dipartimento di Fisica e Astronomia dell'Università degli Studi di Catania, via S. Sofia, 78 - 95123 Catania, Italy
}

Received 12 January 2006 / Accepted 31 March 2006

ABSTRACT

\begin{abstract}
The long-term optical photometry of V711 Tau (HR 1099) from 1975 to 2001 is analysed with different techniques to provide information on the activity of this widely studied RS CVn-type binary. Pooled variance analysis gives a characteristic timescale of evolution for the photospheric pattern of starspots of the order of 100-200 days, while periodogram analysis of seasonal photometric data reveals that the rotational modulation period is $2.837 \pm 0.094 \mathrm{~d}$, without significant changes during the 26 analysed years. Considering subsets of the entire data sequence along which the rotational modulation of the optical flux stays stable, a set of 66 light curves is obtained. They are modelled by means of inversion techniques based on Maximum Entropy and Tikhonov regularizations to derive the distributions in longitude and the total amount of the spotted area on the more luminous K1 IV component that dominates the optical variability of the system. An extensive comparison with published Doppler Imaging maps shows the advantages and drawbacks of inversion techniques based solely on optical photometry. The large-scale structure of the longitudinal distribution of the spotted area is correctly reproduced, although individual starspots revealed by Doppler Imaging cannot be detected because of the limited spatial resolution of the photometric imaging. On the other hand, the variation of the total spotted area is best reconstructed from optical photometry and shows an activity cycle with a period of $19.5 \pm 2.0 \mathrm{yr}$, upon which a short-term modulation might be superposed having variable amplitude and phase and a mean duration of approximately 3-5 years. The distributions of the spotted area versus longitude show the presence of one dominant preferential longitude from 1975 to 1989 ; afterwards, two preferential longitudes with comparable spotted areas are detected. Their migration rates with respect to the orbital reference frame are variable versus time, possibly as a consequence of a variable drift rate of the respective non-axisymmetric dynamo modes. The variation of the orbital period of the system appears to be correlated with the magnetic activity of the K1 IV component. Specifically, the decrease of the orbital period that occurred around 1990 coincides with a re-configuration of the stellar magnetic field, as indicated by the simultaneous variation of its different proxies. In combination with previous studies, these results allow us to discriminate among different theoretical models proposed to explain the connection between magnetic activity and orbital dynamics.
\end{abstract}

Key words. stars: activity - stars: starspots - stars: magnetic fields - stars: late-type - stars: binaries: spectroscopic stars: individual: V711 Tauri - stars: individual: HR 1099

\section{Introduction}

V711 Tauri (HR1099, HD 22468) is one of the most extensively studied members of the RS CVn class of magnetically active close binaries. It is a detached, non-eclipsing SB2 system, consisting of a K1 subgiant star, filling about $80 \%$ of its Roche lobe, and a G5 dwarf star on a 2.837 day orbit (Fekel 1983; Donati 1999). Its apparent visual magnitude is $\sim 5.7$ and its distance is $29.0 \pm 0.7 \mathrm{pc}$ (Perryman et al. 1997). The system is also the primary component of the visual binary ADS 2644 whose secondary component is a $\mathrm{K} 3 \mathrm{~V}$ star $\sim 6^{\prime \prime}$ apart. Owing to an extended outer convection zone and fast rotation enforced by tidal synchronization, the K1 IV star hosts a powerful hydromagnetic dynamo and shows a very high level of solar-like magnetic activity. The G5 V component is also rapidly rotating, but its convection zone is less extended, which makes it much less active than the subgiant. Therefore, the magnetic activity signatures of V711 Tau are dominated by the K1 IV star. They include a rotational modulation in the optical passband with an amplitude up

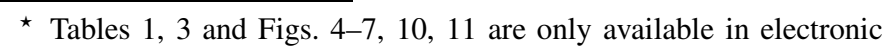
form at http://www. edpsciences.org

$\star \star$ Deceased. to $\sim 0.2$ mag due to cool photospheric spots orders of magnitude larger than sunspots (e.g., Rodonò et al. 1986; Henry et al. 1995). Optical flares have been observed with amplitudes up to $0.4 \mathrm{mag}$ in the $V$ passband, which is quite exceptional for a star more luminous than the Sun (Henry \& Hall 1991; Foing et al. 1994). Long-term time series of optical photometry were recently analysed by, e.g., Henry et al. (1995), Strassmeier \& Bartus (2000), and Alekseev \& Kozhevnikova (2004), who found evidence for a 16 -yr activity cycle and, possibly, for a secondary cycle of $5.5 \mathrm{yr}$. Henry et al. (1995) performed a simple photometric modelling, finding the evolution of individual starspots, their rotation rates, and some indication of preferential longitudes of spot formation. Most of the recent studies of the photosphere of the K1 IV component have been based on Doppler imaging and show a nearly polar spot that has persisted for at least twenty years, although with apparent changes of its finer details (Vogt et al. 1999). Smaller spots appear at lower latitudes and show systematic motions that may be an indication of an anti-solar surface differential rotation (i.e., with the pole rotating faster than the equator, Vogt et al. 1999) or of a complex pattern of surface flows and short-term intrinsic spot evolution, even on time scales of a few days or tens of days (Strassmeier \& Bartus 2000). The recent 
studies by Donati (1999), Donati et al. (2003), and Petit et al. (2004) have provided us with maps of the surface magnetic fields based on the Zeeman and classical Doppler imaging techniques that have confirmed the long-term persistence of the polar spot. Outside the polar spot, the magnetic field topology appears to be dominated by extended regions with a predominantly azimuthal field that is remarkably different from the magnetic topology of the solar photosphere. No evidence of cyclic changes has been found, although the time series of Zeeman Doppler maps has been extended for more than ten years. The differential rotation reported by Petit et al. (2004) is solar-like and with a relative amplitude of $0.7 \%$, i.e., about 30 times smaller than in the case of the Sun. Such a result questions the previous determinations that suggested an anti-solar differential rotation, possibly owing to the intrinsic spot evolution on time scales of a few weeks, and the limited accuracy of some of the previous data sets.

The chromospheric activity of the K1 IV star is very intense, as indicated by the strong time-variable $\mathrm{Ca}$ II $\mathrm{H} \& \mathrm{~K}, \mathrm{H} \alpha$, and ultraviolet emissions (e.g., Rodonò et al. 1987; Dempsey et al. 1996; Robinson et al. 1996). Busà et al. (1999) applied a Doppler imaging technique to the time variable profile of the chromospheric $\mathrm{Mg}$ II $\mathrm{h}$ line and found that a polar active region is present on the K1 IV star with a systematically red-shifted line emission that indicates downward directed motions. The profiles of the chromospheric $\mathrm{H} \alpha$ and $\mathrm{Mg}$ II $\mathrm{h}$ lines in quiescence show evidence of two components possibly arising from active and quiet chromospheric regions, as discussed by Lanzafame et al. (2000). Flares are also frequently detected with $\mathrm{Mg}$ II h extended line wings interpreted as being caused by motions with velocities up to $1000 \mathrm{~km} \mathrm{~s}^{-1}$ (Busà et al. 1999). The observations in the extreme ultraviolet and in the X-ray domains provided evidence for an intensively heated, highly time-variable transition region and coronal structures with a remarkable increase of the emission measure distribution around $\log T(\mathrm{~K}) \sim 6.9$, which has no counterpart in the solar corona, except during the most energetic flares (Sanz-Forcada et al. 2002; Ness et al. 2002). Audard et al. (2001) reported that the X-ray flux is maximum when the $\mathrm{K} 1 \mathrm{IV}$ star is in front, thus confirming that it dominates the coronal emission of the system. Flares in the corona were also detected by radio observations (e.g., Umana et al. 1995), and they may possibly show a cyclic occurrence on time scales of a few months (Richards et al. 2003). The flare activity was best studied by means of co-ordinated multi-wavelength and multisite campains that allow us to follow the time evolution of the high-energy fluxes, thus estimating flare frequency, time scales and energy budget (e.g., García-Alvarez et al. 2003; Osten et al. 2004).

In the present paper, we shall analyse the available optical observations of V711 Tau using the Maximum Entropy and Tikhonov regularized spot models introduced by Lanza et al. (1998a, 2002). The previously analysed time series are now extended by means of new observations reaching a time span of 26 years. This will allow us to find the distribution of the spotted area versus longitude in the photosphere of the K1 IV star and its time variations, thus obtaining information on the activity cycle, the presence of preferential longitudes, and their time evolution.

The starspot models based on extended sequences of wideband optical light curves provide results complementary to those derived by the Doppler imaging techniques that are usually unable to trace the component of the spot pattern uniformly distributed over the disk of the star, the variations of which are important to characterize the activity cycle of the subgiant component. Moreover, V711 Tau is an important system for studying the possible connection between orbital period variation and magnetic activity cycles in RS CVn binaries because the longterm radial velocity monitoring of this system has revealed an orbital period change with a relative amplitude of $\sim 9 \times 10^{-5}$ and has excluded the presence of a light-time effect due to a third body. This result indicates that the orbital dynamics is affected by physical processes internal to the system (Frasca \& Lanza 2005).

\section{Observations}

\subsection{The photometric data set}

To study the optical variability of V711 Tau, we collected its photometry from the literature starting from the compilation by Henry et al. (1995) that goes from 1975 to 1993 . We considered only $V$-band observations because they represent the most complete and homogeneous data set. Henry et al. (1995) provided the differential standard $V$ magnitude with respect to the comparison star 10 Tau (HR1101). They collected and homogenized data from several sources listed in their Table 10 and added new observations obtained by their automated telescopes ${ }^{1}$. We added data for the years 1982-1991 obtained by Mohin \& Raveendran (1993), who also used 10 Tau as a comparison star.

New photometric observations of V711 Tau were obtained by means of the Phoenix-25 APT and the APT-80 of INAFCatania Astrophysical Observatory during the years 1991-2001, again using 10 Tau as a comparison star and 12 Tau as a check star. The photometric precision of the $V$-band data of the Phoenix-25 APT is about $0.01 \mathrm{mag}$, whereas that of the Catania APT-80 is about 0.005 mag. The instrumental equipments and observational strategies of our APTs are described by Rodonò et al. (2000) and Rodonò et al. (2001).

It is important to notice that the photometric accuracy of the observations collected and analysed in the present paper is not constant. It ranges from 0.01-0.02 mag for the data up to 1989 , mostly obtained by manual photometry by different observers and telescopes, to $0.005-0.01 \mathrm{mag}$ for the data since 1990, which were obtained almost exclusively by means of automated photometry with only two telescopes.

All the photometric observations of V711 Tau include the contribution of the close tertiary companion ADS 2644B. It was removed from all the light curves to avoid a dilution of the photometric variations of the close active binary. Specifically, the magnitude difference $\Delta V$ with respect to the comparison 10 Tau was corrected for the third light, according to the equation:

$\Delta V=-2.5 \log \left[10^{-0.4 \Delta V_{\mathrm{obs}}}-10^{-0.4\left(V_{\mathrm{ter}}-V_{\mathrm{cp}}\right)}\right]$,

where $\Delta V_{\text {obs }}$ is the observed magnitude difference of the star with respect to $10 \mathrm{Tau}$, including the third light, $V_{\mathrm{cp}}=4.28$ is the magnitude of the comparison star, and $V_{\text {ter }}=8.83$ that of the tertiary component.

The complete data set of the photometric observations of V711 Tau is plotted versus the time in Fig. 1.

\subsection{Pooled variance and periodogram analysis}

To point out the relevant timescales of variation of our photometric time series, it is useful to apply the pooled variance analysis as proposed by Dobson et al. (1990), Donahue \& Baliunas (1992), and Donahue et al. (1997). In Fig. 2, the pooled variance, i.e., the average variance of the data subsets of length $\tau$,

\footnotetext{
1 See also http://schwab.tsuniv. edu/t3/fourstar/ fourstar.html
} 


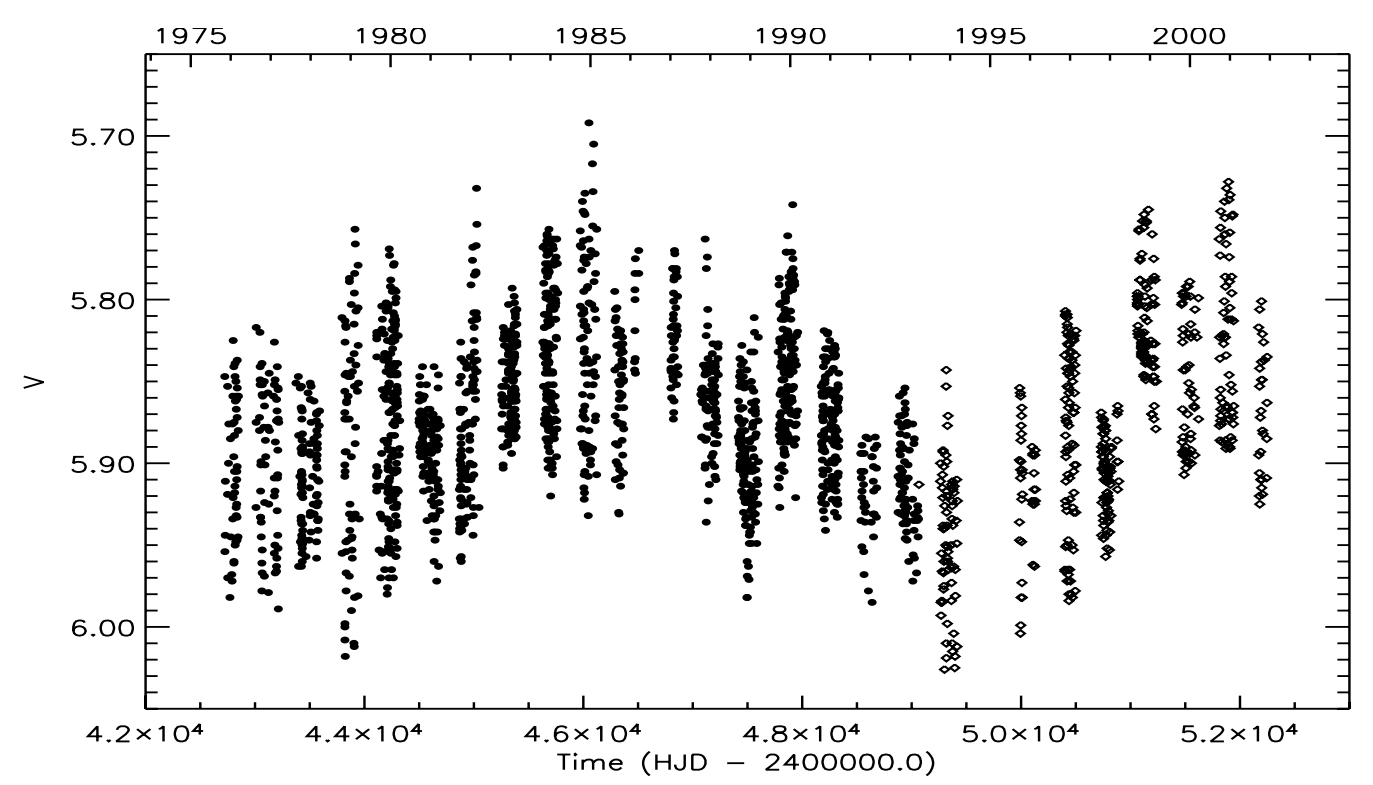

Fig. 1. The $V$-band magnitude of V711 Tau versus time. Literature data (filled circles; Henry et al. 1995; Mohin \& Raveendran 1993), as well as our new APT observations (open diamonds), are plotted. The contribution of the light from ADS 2644B was removed as explained in the text.

is plotted versus $\tau$, allowing us to identify three characteristic time intervals. For timescales shorter than about $1 \mathrm{~d}$ (interval A in Fig. 2), the variance of the data mainly arises from the errors of the photometric observations that are of the order of $\sim 0.01 \mathrm{mag}$. Flaring activity on time scales of a few hours and with amplitudes $>0.1 \mathrm{mag}$, although sometimes observed, is not so frequent as to significantly affect the variance in this range. For timescales longer than $1 \mathrm{~d}$, the rotational modulation of the flux due to the active regions unevenly distributed in longitude produces a rise of the pooled variance up to $\tau \sim 3 \mathrm{~d}$, corresponding to the rotation period of the component stars. The pooled variance stays more or less constant for time intervals up to $\tau \sim 200 \mathrm{~d}$ (interval B) that should correspond to the lifetime of the largest surface brightness inhomogeneities (Donahue et al. 1997), i.e., the complexes of spot activity in the photosphere of the K1 IV star, which dominates the light variation of the system because it is about 4 times more luminous than the G5 V star. For timescales longer than about $200 \mathrm{~d}$, the intrinsic evolution of the large surface structures dominates the light variation up to timescales of the order of a decade when the long-term variation is due to the activity cycle (interval $\mathrm{C}$ ). The apparent increase of the variance fluctuations at the longest timescales is due to the small statistics, i.e., to the limited number of intervals over which the pooled variance is computed.

A search for periodic signals was performed for each season of observation by means of the Scargle-Lomb periodogram to look for possible systematic changes of the period of the rotational modulation versus time as observed in, e.g., II Peg (Rodonò et al. 2000) and in some young rapidly rotating stars (Messina \& Guinan 2003). The pooled variance analysis indicates that the lifetimes of large activity complexes dominating the flux modulation are of the order of $200 \mathrm{~d}$. Therefore, it is advisable to look for changes of the period of the rotational modulation considering data subsets no longer than about 200 d, i.e., data within the same observing season. Twenty-six seasons of data were analysed according to the prescriptions by Horne \& Baliunas (1986) going from 1976 to 2001, and a single, highly significant periodicity was detected within each season (i.e., with a confidence level larger than $99.9 \%$ ), pratically coincident with the orbital period. However, no significant variation of such a

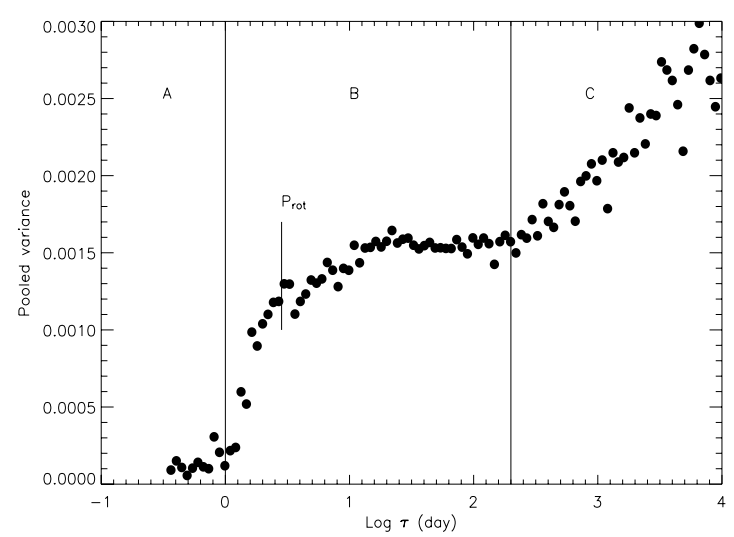

Fig. 2. The pooled variance of the $V$-band photometric data versus the timescale. Three main ranges of variation can be identified and are labelled as A, B, and C, respectively. The timescale corresponding to the rotation period $\left(P_{\text {rot }}\right)$ is marked by a vertical segment.

rotational modulation period from season to season was detected, yielding an average rotation period equal to $2.837 \pm$ $0.094 \mathrm{~d}$, over the years 1976-2001.

\subsection{Preparation of the light curves}

To model the light variations of V711 Tau with a surface distribution of cool spots, we subdivided the whole data set into individual light curves. The division was made, as always, by selecting time intervals with a stable flux modulation, i.e., avoiding significant differences (i.e., larger than $\sim 0.05$ mag) between observations falling close in phase to each other. Moreover, individual observations more than about 0.05 mag apart from the average modulation of a given light curve were discarded as they were probably due to short-term flaring activity. Data were put in phase by means of Fekel (1983) ephemeris:

$H J D_{\text {conj }}=2442766.08+2.83774 \times E$,

where the epoch of conjuction is the one with the K1 IV star in front of the G5 V star. As a matter of fact, the orbital period of 
V711 Tau is not constant on timescales of decades. Therefore, a correction was applied from 1989.93 on to put the light curves in phase with the actual orbital motion of the system, as indicated by the radial velocity curves (see Frasca \& Lanza 2005).

The ellipticity effect due to the tidal distortion of the close component stars of V711 Tau was not removed because our light curve model can account for it as well as the gravity-darkening and the reflection effects (see Sect. 3).

Following this approach, 66 light curves were constructed ranging from mid 1975 to the end of 2001, including a total of 2377 photometric points. They are listed in Table 1 that includes, in its first to eighth columns, respectively, the mean epoch of the light curve (year), the epochs of its initial and final observations, its mean epoch (HJD), the number of observations $M$, the diameter of the telescope used $D_{\mathrm{T}}$, the photometric system $P(\mathrm{~S}$ : Johnson $V$-band, St: Strömgren $y$-band) and the original reference of the observations. No conversion was needed for the few Strömgren $y$-band observations to the $V$-band of the Johnson's system because the correction was significantly smaller than the accuracy of the observations (Olsen 1983).

The brightest $V$ magnitude of our light curve sequence was $5.692 \pm 0.008$, corrected for the third light, in the 1985 season at an orbital phase of 0.1178 . This value is assumed as the magnitude of the unspotted system, thus establishing the magnitude scale calibration for our light curve modelling.

\section{Spot modelling technique}

The reconstruction of the brightness distribution over the surface of a star by modelling the rotational modulation of wide-band optical fluxes is an ill-posed problem. The modulation provides information only on the variation of the projected spot area versus the rotational phase, i.e., the stellar longitude. Since V711 Tau is not an eclipsing system, it is not possible to take advantage of the scanning made by one component star over the disk of the other during eclipses to improve the photometric spot modelling (cf., e.g., Lanza et al. 1998a, 2002).

In principle, it is possible to determine a unique spot distribution by minimizing the $\chi^{2}$ of the residuals between the observed and the model light curve, but such an approach is unsatisfactory because the solution is unstable, i.e., small variations of the input data lead to large changes of the spot map. This is due to the role played by the noise in the $\chi^{2}$ minimization, implying that most of the structures appearing on the spot map actually come from the overfitting of the light fluctuations produced by the star intrinsic variability or measurement errors.

It is possible to overcome the uniqueness and stability problems by introducing a regularizing function into the solution process. This corresponds to the a priori assumption of some specific statistical property for the spot map, which allows us to select one stable map among the potentially infinite number of maps that can fit a given light curve. The two most used a priori assumptions are the Maximum Entropy (hereinafter ME; Gull \& Skilling 1984; Vogt et al. 1987) and the Tikhonov (hereinafter T; Piskunov et al. 1990) regularizations. They are particularly suited when the spot map consists of an array that specifies the spot covering factor in each surface element (or pixel) of the model star. The covering factor $f$ gives the specific intensity $I$ of each surface element according to the definition: $I=(1-f) I_{\mathrm{u}}+f I_{\mathrm{s}}$, where $I_{\mathrm{u}}$ is the specific intensity of the unspotted photosphere of the surface element, $I_{\mathrm{S}}$ that of the spotted photosphere, and $0<f<1$.

A detailed description of the application of the ME and T regularizations to spot modelling problems was presented by
Lanza et al. (1998a) to whom we refer the reader for a detailed discussion of our approach. Here we only recall that the ME spot maps are computed by a constrained minimization of the functional:

$Q_{\mathrm{ME}}=\chi^{2}-\lambda_{\mathrm{ME}}\left(S_{1}+S_{2}\right)$,

whereas the T maps are computed by the constrained minimization of:

$Q_{\mathrm{T}}=\chi^{2}+\lambda_{\mathrm{T}}\left(T_{1}+T_{2}\right)$

where $\chi^{2}$ is the normalized sum of the squared residuals between the observed and the synthetized light curves, $S_{k}$ is the entropy of the spot map of the $k$ th $\operatorname{star}(k=1,2), T_{k}$ is the T functional of the spot map of the $k$ th star, and $\lambda_{\mathrm{ME}}$ and $\lambda_{\mathrm{T}}$ are the Lagrange multipliers for the $\mathrm{ME}$ and the $\mathrm{T}$ regularizations, respectively. The explicit expressions for $\chi^{2}, S_{k}$, and $T_{k}$ and the procedure of evaluation of the Lagrange multipliers are described by Lanza et al. (1998a).

The synthesized light curve for a given distribution of the covering factor is computed using the code introduced by Lanza et al. (2002). It adopts Roche geometry to describe the surfaces of the components. The photosphere of each component is subdivided into squared elements of side $s_{\mathrm{e}}=1^{\circ}$ to warrant a relative precision of the order of $10^{-4}$ in the numerical evaluation of the flux that emerges from the stellar disks. The flux from each surface element is computed by adopting Kurucz (2000) atmospheric models with a linear limb darkening law and taking into account gravity darkening effects, according to Kopal (1959). The reflection effect is treated assuming blackbody reirradiation with given bolometric albedo and Lambert's law for the angular distribution of the re-emitted radiation. Small errors in the atmospheric models only give rise to second-order effects in our analysis of stellar activity because we are mainly interested in the differential variations of the spot pattern properties from season to season. Absolute properties of the spots cannot be extracted from single-band data because systematic errors arise, among others, from the unknown unspotted light levels of the system components and the assumption of single-temperature spots. Specifically, the brightest magnitude of V711 Tau ever observed is probably higher than the truly unspotted magnitude because spots may always be present on the photosphere of its active component star.

\section{Model parameters}

In our spot maps we always adopted squared map elements of side $s=18^{\circ}$, whereas the fluxes were computed with the finer subdivision $s_{\mathrm{e}}=1^{\circ}$. The orbital parameters, the masses, the radii, and the effective temperatures of the component stars were derived by Donati (1999). The luminosity ratio of the two components in the $V$-band was computed assuming an unspotted magnitude of 5.80 and 7.20 for the K1 IV and the G5 V stars, respectively (Donati 1999). The spot temperature deficit was assumed to be $1000 \mathrm{~K}$ for both stars (see also Strassmeier \& Bartus 2000), an average value for the active components of RS CVn binary systems (cf., e.g., Eaton 1992).

The values of the Roche potential $\Omega$ at the surface of each star, as well as the relative radii at the point (i.e., along the line joining the centres of the components), side, back, and pole for each component are listed in Table 2, respectively.

The photospheric models by Kurucz (2000) were used to evaluate the ratio of the specific intensities of the spotted and 
Table 2. Geometrical and physical parameters adopted for modelling the V711 Tau light curves.

\begin{tabular}{|c|c|c|c|}
\hline \multicolumn{3}{|c|}{ Orbital elements of V711 Tau } & \\
\hline \multicolumn{2}{|l|}{ Element } & \multicolumn{2}{|l|}{ Ref. $\left({ }^{a}\right)$} \\
\hline Semi-major axis & 1.00 & & \\
\hline Eccentricity & 0.00 & 1 & \\
\hline Inclination (deg) & 38.0 & 1 & \\
\hline Period (day) & 2.83774 & 1 & \\
\hline \multicolumn{4}{|c|}{ Stellar and model parameters } \\
\hline Stellar parameter & G5 V & K1 IV & Ref. $^{a}$ \\
\hline Roche potential $(\Omega)$ & 10.625 & 3.862 & 1,2 \\
\hline Fract. radius (point) & 0.1070 & 0.3590 & 1,2 \\
\hline Fract. radius (side) & 0.1067 & 0.3347 & 1,2 \\
\hline Fract. radius (back) & 0.1070 & 0.3483 & 1,2 \\
\hline Fract. radius (pole) & 0.1066 & 0.3241 & 1,2 \\
\hline Mass $\left(M_{\odot}=1\right)$ & 0.8 & 1.0 & 1 \\
\hline$V$-band fractional luminosity & 0.216 & 0.784 & 1 \\
\hline Bolometric correction (mag) & -0.10 & -0.50 & 1,3 \\
\hline Effective temperature $(\mathrm{K})$ & 5500 & 4750 & 1 \\
\hline Surface gravity $\log g\left(\mathrm{~cm} \mathrm{~s}^{-2}\right)$ & 4.26 & 3.30 & 1 \\
\hline Starspot temperature $(\mathrm{K})$ & 4500 & 3750 & 4 \\
\hline Gravity darkening & 0.25 & 0.25 & 5 \\
\hline$u_{V}$ (unspotted) & 0.734 & 0.792 & 6,7 \\
\hline$u_{V}($ spotted $)$ & 0.798 & 0.823 & 6,7 \\
\hline$C_{\mathrm{s}}$ & 0.319 & 0.161 & 6 \\
\hline Bolometric albedo & 1.00 & 0.30 & 5 \\
\hline Albedo in the $V$ pass-band & 1.01 & 0.27 & 2,5 \\
\hline
\end{tabular}

${ }^{a}$ References: [1]: Donati (1999); [2]: Kopal (1959); [3]: Flower (1996); [4]: Eaton (1992); [5]: Eaton et al. (1993); [6]: Kurucz (2000); [7]: DiazCordoves et al. (1995).

unspotted photospheres at the disk centre $\left(C_{\mathrm{s}}\right)$ and the linear limb-darkening coefficients in the $V$-band $\left(u_{V}\right)$. Systematic errors due to the linear limb-darkening approximation were reduced by means of the least square procedure devised by Diaz-Cordoves et al. (1995) for Kurucz's models.

The adopted gravity darkening coefficients are appropriate for stars with outer convective envelopes. The bolometric albedo for the late-type K1 IV component was assumed to be smaller than unity in consideration of its extended convective envelope (cf., e.g., Eaton et al. 1993). All model parameters we adopted to compute the light curve fits are listed in Table 2, together with the corresponding references.

\section{Results}

\subsection{Light curve best fits and spot maps}

The best fits of the light curves obtained with the ME regularizing criterium are always good and are shown in Figs. 3-7. We assume a standard deviation of the observations equal to $0.02 F_{0}$, where $F_{0}$ is the unperturbed value of the flux of the system in the $V$ passband at quadrature, and a constant value of the Lagragian multiplier $\lambda_{\mathrm{ME}}=4.0$. The values of the $\chi^{2}$ of the ME best fits are listed in Table 3 . Very similar best fits are obtained by means of the T regularizing criterium, adopting a constant value of the Lagrangian multiplier $\lambda_{\mathrm{T}}=48.0$ up to 1991.12 and $\lambda_{\mathrm{T}}=300.0$ from 1991.89 to 2001.80; their $\chi^{2}$ values are listed in Table 3 as well, but the light curve best fits are not plotted. Note that $\chi^{2}$ values greater than 1.0 are due to the short-term light fluctuations of the system that particularly affect those light curves whose observations span several rotations. Values of $\chi^{2}$ signifi- cantly lower than 1.0 do not indicate an overfit, but are due to the lower intrinsic stellar fluctuations and the higher accuracy of the photometric data, in particular of the subsets after 1989 obtained by means of automated photometry (cf. Sect. 2.1).

A sample of the distributions of the spot covering factor on the K1 IV star is provided by Fig. 8, which shows some spot maps in orthographic projection for an observer viewing the star from the North Pole adopting the same reference frame as Vogt et al. (1999). It is interesting to compare the photometric ME maps for the 1985.72, 1989.77, and 1992.78 light curves with the ME Doppler Imaging (hereinafter DI) maps published by Vogt et al. (1999) for the mean epochs 1985.86, 1989.73 (and 1989.79), and 1992.83, respectively. These DI maps were thresholded by means of photometric data, making our comparison more meaningful than that with other DI maps based on line profile data alone. Our technique proves capable of reconstructing the large-scale features of the longitudinal distribution of the filling factor, as is expected, given that the spot visibility is modulated by stellar rotation. On the other hand, the information on the spot latitudinal distribution derived from wide-band light curves is very poor, as is indicated by the lack of agreement between the latitudinal distributions of the spot covering factor obtained with our modelling technique and those given by DI. Our ME maps show spots always located in the Northern hemisphere, whereas the DI maps sometimes show small spots in the Southern hemisphere (note that the limbs of the projected disks correspond to the equator in our maps and to the latitude $30^{\circ} \mathrm{S}$ in Vogt et al.'s maps). Moreover, the details of the spot pattern revealed by DI are not reproduced in our maps showing that our technique does not resolve individual spots on the active component of V 711 Tau. Some details in our ME maps are due to the so-called super-resolution effect, which is related to the a priori assumptions introduced by the ME regularization, as was explained by Lanza et al. (1998a). Similar results are obtained by comparing the other photometric ME maps of our sequence with the DI maps of Vogt et al. (1999), Donati (1999), and Petit et al. (2004), the latter two sequences being based on line profile data alone. Only for those few epochs when the light curve amplitude is smaller than $\sim 0.05-0.07 \mathrm{mag}$ and/or the spot pattern is varying on a timescale smaller than the extension of the data set, there are significant differences in the longitudinal distribution of the spotted area between photometric and DI maps. This is due to the fact that photometric mapping is poorly constrained by the data in those circumstances so that the a priori assumptions introduced by the regularizing criteria strongly affect the reconstructed spot distribution.

The spot maps based on the T regularization give similar longitudinal distribution of the spot pattern (see below), giving further support to the results obtained by the ME maps. The latitudinal distribution is less concentrated, with a significant spot occupacy also in the latitude belts South of the equator. This is clearly due to the influence of the $\mathrm{T}$ regularizing criterium that dominates the latitudinal distribution, given the absence of enough latitudinal information in wide-band photometric data.

Simultaneous ME and T spot maps of the less luminous G5 V star are significantly different, showing the impact of the different a priori assumptions of the two regularizing criteria on the reconstructed spot distribution. This is indeed expected, given that the luminosity of the G5 V star is only $\sim 1 / 4$ of that of the K1 IV star which dominates the light curve variations of the system. Therefore, we shall not discuss the spot maps of the G5 V star further because their information content is too limited, and we shall only consider the spot distribution on the K1 IV star that is much better constrained by our data set. 


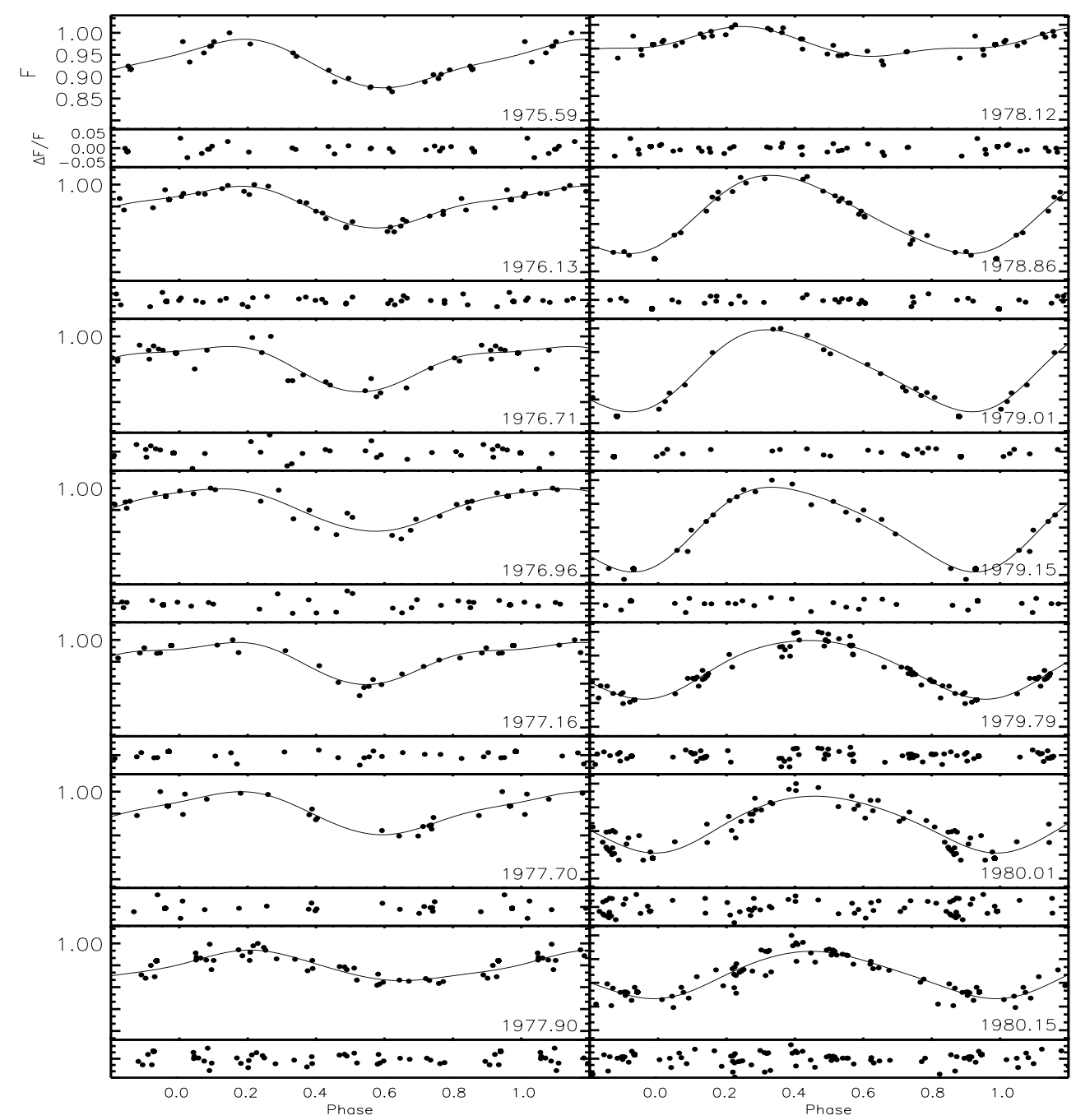

Fig. 3. Upper panels: the $V$-band light curves of V711 Tau from the 1975 season to the first half of the 1980 season (filled circles) with the corresponding ME best fits (solid lines); the label identifying each light curve is indicated in the corresponding plot; the flux is normalized to the maximum observed value in each light curve. Lower panels: the relative differences between the observed $V$-band flux and the corresponding best-fit values obtained with our ME model.
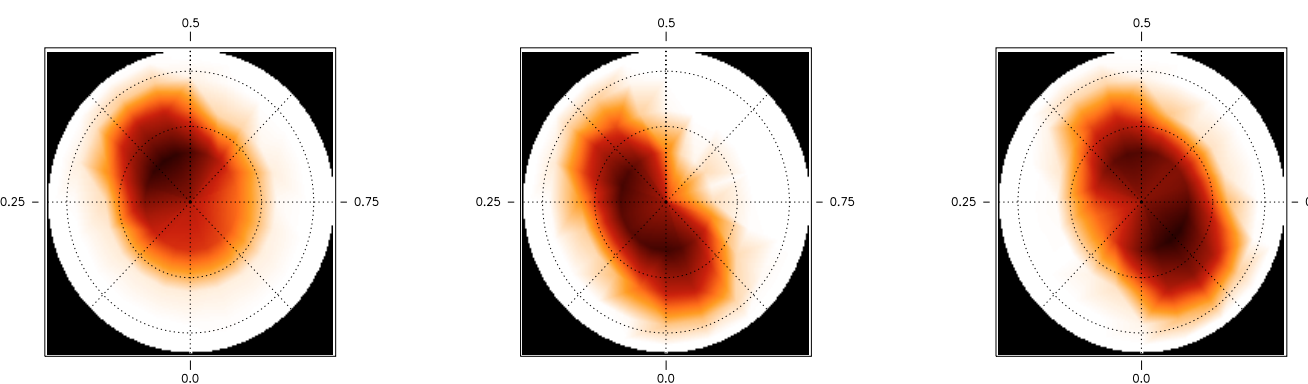

Fig. 8. The distribution of the spot coverage factor $f$ over the surface of the K1 IV component star for the ME best-fit map of the 1985.72 (left panel), the 1989.77 (middle panel) and the 1992.78 (right panel) light curves, respectively. The limb of the stellar disk is the projection of the equator, and the dotted circles mark the latitudes $30^{\circ} \mathrm{N}$ and $60^{\circ} \mathrm{N}$, respectively. The positions of the central meridian of the stellar disk, as seen by an observer on the Earth at the labelled phases, are indicated by the small ticks.

\subsection{Longitude distribution and total area variation of the spot pattern}

It is important to notice that the spot maps obtained with our approach must be considered as an intermediate product of the light curve analysis. The final results are represented by the distributions of the spotted area versus longitude and the variation of the total spotted area versus time because these quantities are not strongly affected by the assumed unspotted light level and the a priori assumptions of our regularizing criteria. In Figs. 911 , the distributions of the spotted area versus longitude derived from the ME and T light curve models are plotted for the K1 IV component. On these plots, the longitude is measured from the substellar point in the direction opposite to the orbital motion, so that the phase at which a given surface element crosses the central meridian of the stellar disk is equal to its longitude. This 


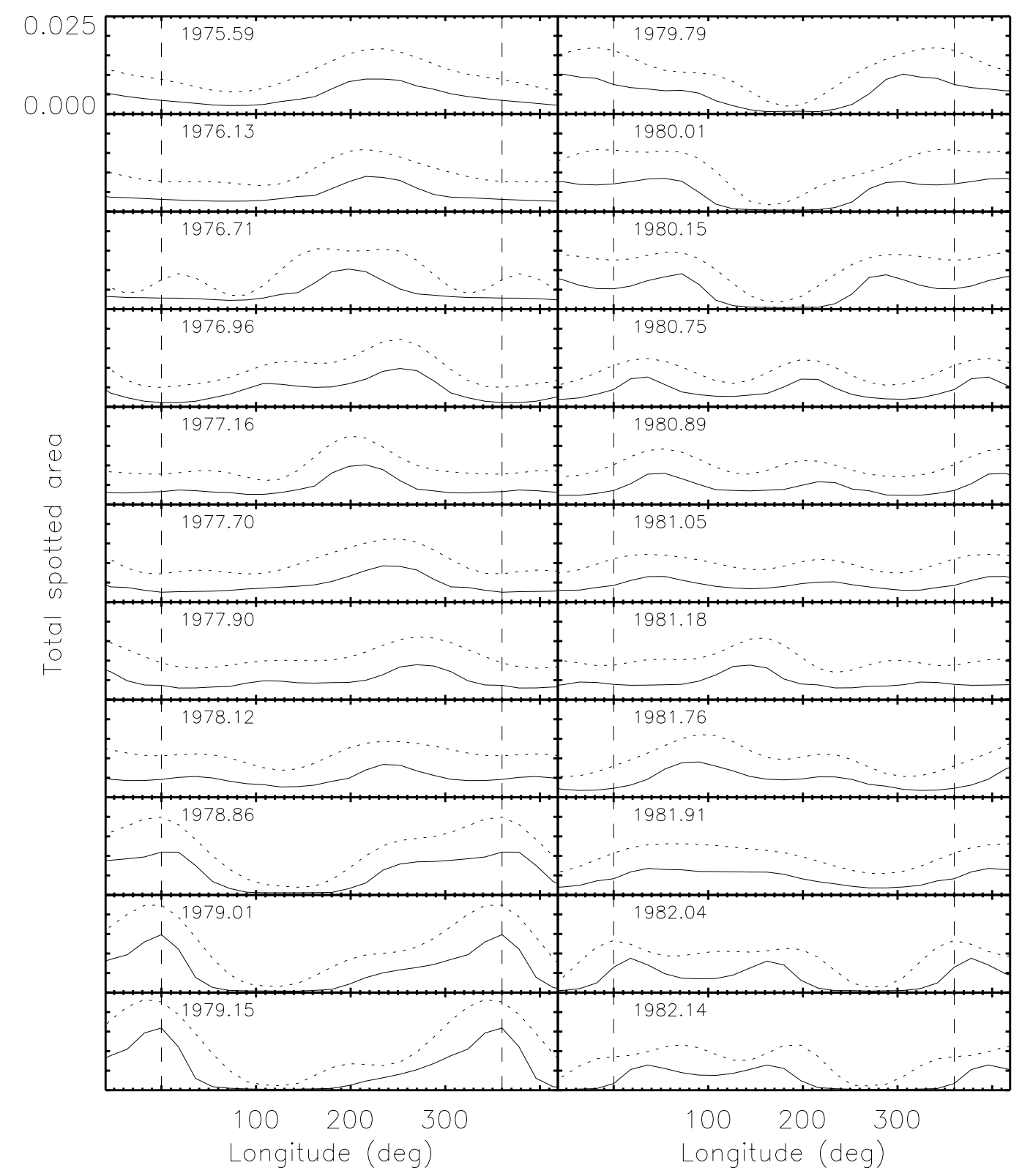

Fig. 9. The distribution of the spotted area vs. longitude on the K1 IV star for the light curve models from 1975.59 to 1982.14 obtained with the ME regularization (solid lines) and with the T regularization (dotted lines), respectively. The area unit is the photospheric area of the star.

allows us a direct comparison of the spot distributions with the flux variations of the individual light curve fits (cf. Figs. 3-7).

The distributions obtained with the ME and $\mathrm{T}$ regularizing approachs are remarkably similar, although the filling factors derived with the $\mathrm{T}$ criterium are systematically larger than those derived with the ME criterium. This is a well-known effect related to the tendency of ME to minimize spot occupacy on the photosphere of a star, whereas the T criterium yields larger spot filling factors because it tends to smooth out the surface brightness variations (cf., e.g., Lanza et al. 1998a). The statistical errors of the spot relative area in each $18^{\circ}$ longitude bin are between $10 \%$ and $30 \%$. The low inclination of the rotation axis of the active $\operatorname{star}\left(i=38^{\circ}\right)$ makes it possible to account for the variation of the mean light level from season to season by changing the area of the high-latitude spot pattern, which is always in view. Therefore, there is no need for a sizeable component of the spotted area uniformly distributed in longitude at low latitudes that was typical of the spot models of eclipsing binary systems (cf., e.g., Lanza et al. 1998a, 2001, 2002).

When our distributions of the spotted area vs. longitude are compared with DI maps, one must keep in mind the intrinsic limitations of the information that can be extracted from wide-band light curves and line profile distortions, respectively. Specifically, the limited longitude resolution of the maps derived by light curve fitting does not allow us to resolve spots that are closer than about $70^{\circ}-90^{\circ}$ in longitude, even with a photometric precision of a fraction of a hundredth of magnitude. On the other hand, DI techniques are capable of reconstructing the positions and shape of individual spots, but their intensities, i.e., filling factors, are ill-defined unless a best fit to simultaneous photometric data is used to constrain them (cf., e.g., Donati et al. 1992; Vogt et al. 1999). Moreover, DI is basically insensitive to a uniform or slowly varying background of spots on the photosphere of the star. These effects produce a systematic difference 
between the total spotted area estimated from DI and that derived from wide-band light curve fitting, the latter usually being larger (cf., e.g., Donati et al. 2003).

In view of such considerations, it is interesting to compare the DI maps obtained for V711 Tau with our longitudinal spot area distributions limiting the comparison to nearly simultaneous data sets, i.e., differing by no more than $0.1 \mathrm{yr}$ in their mean epochs, because the spot pattern on V711 Tau can significantly change on time scales as short as $2-4$ weeks (Petit et al. 2004). Considering the DI maps in the literature, we performed 24 comparisons with the following results:

i) When the spot area distribution derived from photometry shows only one relative maximum, it coincides within $10^{\circ}-30^{\circ}$ with the longitude of maximum spottedness, as derived from the DI maps, although the overall filling factors are usually different. In this case, simple spot configurations, dominated by an asymmetric polar spot, are usually observed in the DI maps (cf., e.g., the 1985.86 and 1991.10 maps of Vogt et al. (1999) with our 1985.72 and 1991.12 distributions, respectively);

ii) When two relative maxima are present in the longitude distribution of the spotted area, two main cases are found: a) the highest maximum of the distribution usually coincides with the longitude of maximum spottedness in the DI map, whereas the lower maximum coincides with some low latitude spot or an appendage of the polar spot, although not every individual spot has a corresponding maximum in the area distribution; b) the highest maximum corresponds to the longitude of maximum spottedness, whereas the lower one falls in between two main spots. Examples of case a) are provided by comparing, e.g., the 1987.08 map of Vogt et al. (1999) with our 1987.10 distribution, which shows maxima corresponding to spot $\mathrm{B} 2$ and $\mathrm{D}$, but no relative maximum corresponding to spot C; or the map of 1992.94 of Donati (1999) with our 1992.92 distribution showing two relative maxima corresponding to the two main polar spot appendages in the DI map. Examples of case b) are given by comparing, e.g., the DI map of 1996.99 of Donati (1999) with our 1997.05 distribution or the map of Petit et al. (2004) for the epoch 2000.14 with our 2000.07 distribution.

The total spotted area over the surface of the K1 IV star is plotted vs. time in Fig. 12 for the ME and the T models, respectively. The variations are very similar, although the $\mathrm{T}$ criterium gives systematically larger total spotted areas than the ME criterium, as discussed above. A periodogram analysis was performed using the prescriptions of Horne \& Baliunas (1986) and the ME and $\mathrm{T}$ total spotted areas were found to vary with the same periodicity of $19.5 \pm 2.0 \mathrm{yr}$, both with a confidence level larger than $99.9 \%$. A secondary periodicity of $7.8 \mathrm{yr}$ was found for the variation of the ME total spotted area, but it does not appear to be significant since it has a false-alarm probability of $\sim 50 \%$.

The spot area distributions show the presence of preferential longitudes whose positions change versus time. A plot of the longitudes of the relative maxima of the spotted area distribution vs. time is shown in the upper panel of Fig. 13, indicating a quite regular migration of the active longitudes. The variation of the migration rate is apparently correlated with the variation of the total spotted area on the K1 IV component that is shown in the middle panel of Fig. 13. Specifically, a dominant active longitude has been present up to 1987, when a rapid and sharply peaked variation of the total spotted area took place on a timescale of 2-3 years, followed by the growth of a second active longitude on the opposite hemisphere of the star and by

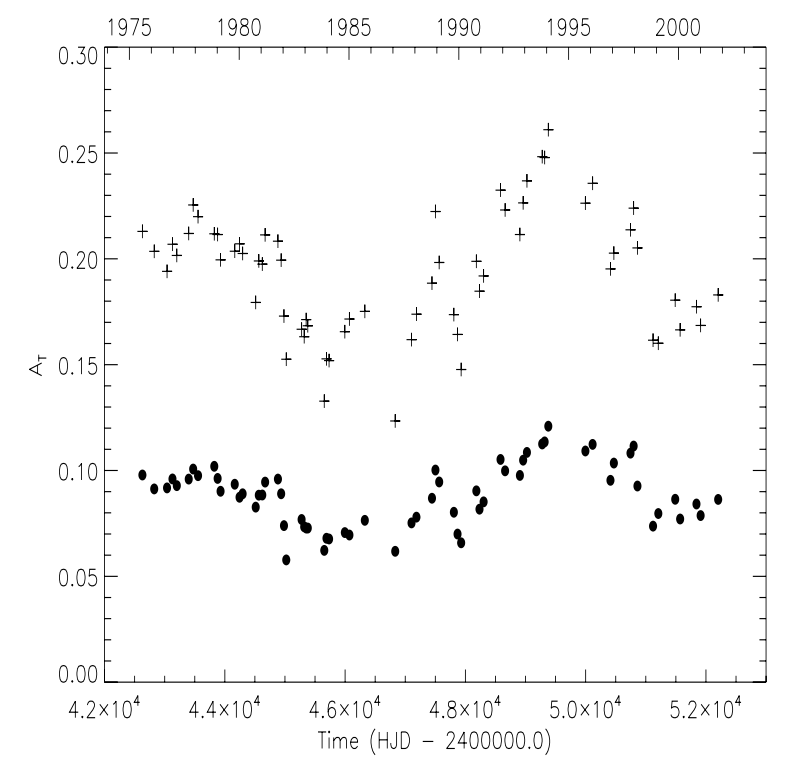

Fig. 12. The total spotted area (in fraction of the photosphere) of the $\mathrm{K} 1 \mathrm{IV}$ star vs. time for the ME (filled circles) and the T solutions (crosses).

a change of their migration rates. It is important to notice that our longitude reference frame is fixed with respect to the orbital motion of the system because we corrected for the effects of the orbital period change on the phase of the light curve. Therefore, the dominant active longitude was migrating toward decreasing orbital phases, i.e., it was rotating faster than the synchronous rotation rate, during the 1976-1988 period, whereas it was migrating toward increasing orbital phases, i.e., it was rotating slower than the synchronous angular velocity, after 1990. Its migration rate with respect to the synchronous rotation was of about $0.112 \mathrm{deg} /$ day during the $1976-1983$ period; it was almost zero during the 1984-1992 period and became about $-0.022 \mathrm{deg} / \mathrm{day}$ during the 1993-2001 period. The second active longitude was sporadically observed during the 1976-1987 period, when it was located on the opposite hemisphere with a significantly smaller spotted area than the dominant one, but with a similar migration rate. It became comparable in area to the previously dominant active longitude in 1989, after the burst of activity that was clearly evident in the variation of the total spotted area, and showed a migration rate of about $-0.040 \mathrm{deg} / \mathrm{day}$ that led to a systematic increase of the distance between the two active longitudes.

\subsection{Orbital period variation}

The orbital period variation of V711 Tau was monitored by Frasca \& Lanza (2005) and their O-C diagram is reproduced in the lower panel of Fig. 13 to allow a comparison with the simultaneous migration of the active longitudes and the variation of the total spotted area. A correlation is apparent between the decrease of the orbital period started around 1990, the burst of spot activity in 1987-1990, and the re-arrangement of the active longitudes on the surface of the K1 IV star. In our opinion, the temporal coincidence between these different phenomena is not a chance, but supports the suggested connection between stellar magnetic activity and orbital period modulation in RS CVn binary systems (e.g., Lanza et al. 1998b; Lanza \& Rodonò 2004). 


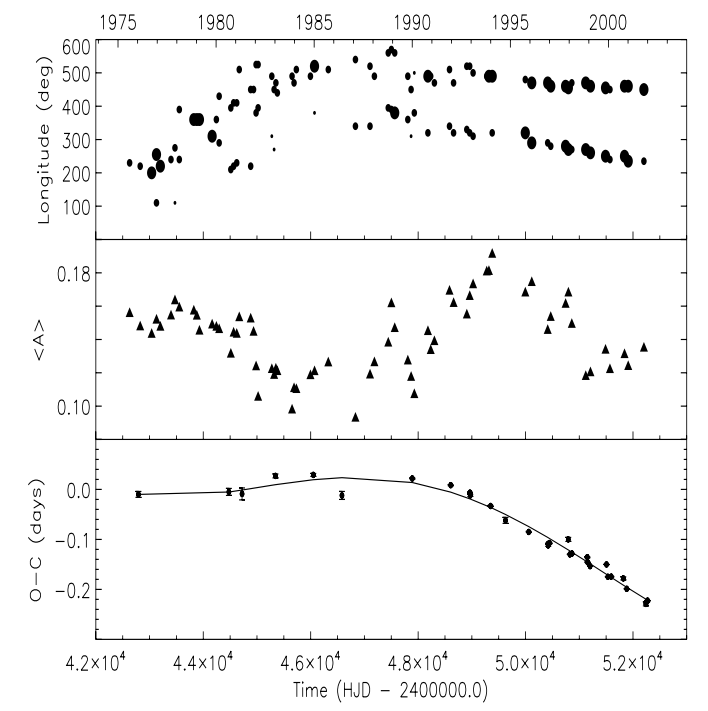

Fig. 13. Upper panel: the longitudes of maximum spottedness, derived by an average of the values of the ME and T distributions, versus time. The dimensions of the symbols are proportional to the values of the maxima in the corresponding spot area distributions. Middle panel: the total spotted area on the K1 IV component versus time, as derived from an average of the ME and T total spotted areas (the area unit is the photosphere of the star). Lower panel: the O-C diagram of HR 1099 showing the difference between the observed epochs of superior conjuctions and those computed with the Fekel's ephemeris (filled circles); the solid line is a fourth-order polynomial best fit to the observed $\mathrm{O}-\mathrm{C}$ values versus time (after Frasca \& Lanza 2005).

\section{Discussion}

We have shown that a pattern of spots with a uniform and constant temperature is adequate to fit all the $V$-band light curves obtained from the long-term photometry of V711 Tau. Our longitude distributions of the spotted area and the time variation of the total spotted area on the K1 IV component appear to be largely independent of the specific regularizing functional we have adopted. The comparison of the longitude distributions of the spotted area with nearly simultaneous DI maps derived with the same regularizing approach reveals that the active longitudes obtained from light curve modelling correspond to actual longitudinal concentrations of photospheric active regions, although the latitudinal distribution of the spot covering factor is not reproduced.

It is interesting to note that two active longitudes separated by $150^{\circ}-180^{\circ}$ are systematically reconstructed for the data sets after 1991, whereas they are only occasionally found in the previous period (e.g., in 1980.75, 1985.01, 1987.10, 1988.77, and 1989.10). They are found not only in the ME solutions, but also in the T solutions, i.e., in the smoothest possible maps compatible with an adequate fit of the data, with the possible exceptions of the 1998.13, 2000.07, and 2001.80 maps where an extended distribution of spots with a single relative maximum is found in the T maps.

Two active longitudes are needed to accurately reconstruct the asymmetric shape of the broad minimum shown by some of the light curves, in particular in the most recent years. As a matter of fact, increasing the spot area at a single localized longitude cannot fit a deep and broad minimum because of the low inclination of the stellar rotation axis that makes the photometric effect of low and intermediate latitude spots significanty smaller than that of the high latitude $\left(>60^{\circ}\right)$ spots. Therefore, a distribution of the spotted area well extended in longitude is required to fit those light curves. It is important to note that the low inclination of the rotation axis makes high latitude spots visible for the whole stellar rotation, thus reducing the photometric constraints on their reconstruction. In other words, the actual longitudinal resolution of our mapping technique is a function of spot latitude, as well as of the accuracy of the photometric data set.

To estimate such a resolution, we performed an extensive analysis of simulated light curve sequences. They were obtained by assuming two spots with an area comparable to the average active longitudes in Fig. 11 put at the same latitude and with a variable longitudinal separation. A pseudo-random Gaussian noise of standard deviation $\sigma$ was added to simulate the effects of the limited photometric accuracy. The number of points in each simulated light curve was fixed at 15 , i.e., comparable with the smallest number of data points in our observational subsets.

The results indicate that the longitudinal resolution of our modelling technique, as indicated by the proper reconstruction of the two assumed active longitudes, is about $160^{\circ}$ at a latitude of $75^{\circ}$ and $130^{\circ}$ at a latitude of $60^{\circ}$ for $\sigma=0.02 \mathrm{mag}$, i.e., the standard deviation of the less accurate photometric data sets. Therefore, the lack of a second well-resolved active longitude in most of the reconstructed spot distributions before 1990 and the systematic appearance of two active longitudes after 1991 are not artefacts due to an insufficient photometric accuracy of the data or the low constraints on the reconstruction of high-latitude spots.

We have also checked the impact of wrong estimations of the proximity effects in producing spurious active longitudes. We found that they can give rise to fixed spurious longitudes, but cannot reproduce the regular migration of the longitudes vs. time that is seen in our spot maps since 1990.

We conclude that, although the longitude of individual spots found by the DI techniques cannot be determined from light curve inversions, our maps display the overall longitudinal distribution of the active regions on the photosphere of the K1 IV star. Our simulations indicate that the minimum longitudinal resolution ranges from $\sim 90^{\circ}$ for spots up to $45^{\circ}$ of latitude North to about $\sim 130^{\circ}-150^{\circ}$ for those at latitudes equal or higher than $\sim 60^{\circ}$.

These results are of particular interest in consideration of the fact that DI maps can be obtained only for late-type stars that rotate quite rapidly $\left(v \sin i>20-25 \mathrm{~km} \mathrm{~s}^{-1}\right)$. For slowly rotating stars, only light curve inversion can be used to study the distribution of the active regions on their surface. The application of $\mathrm{ME}$ and $\mathrm{T}$ regularizing techniques to the light curve inversion problem allows us to derive substantially unique and meaningful results useful for a study of the active longitudes and activity cycles on such stars. Simple light curve modelling based on two or three circular spots does not possess such uniqueness and may sometimes be grossly in error, as shown by the comparison made by Vogt et al. (1999).

The mapping of the photosphere of the K1 IV component based on simultaneous line profile and wide-band photometry inversion has sometimes shown the presence of spots $~ 300-$ $500 \mathrm{~K}$ hotter than the unperturbed photosphere (Donati et al. 1992; Vogt et al. 1999), specifically during the 1988 and 1989 observing seasons that corresponded to a period of particularly intense activity (cf. Fig. 12). The presence of facular structures at several epochs is also suggested by the analysis of the $B-V$ vs. $V$-magnitude variations presented by Aarum-Ulvas \& Henry (2004). It is important to notice that the temperature of the spots cannot be derived from single passband data, such as those analysed in the present paper. Therefore, the introduction of a combination of cool and hot spots in our model would produce 
non-unique and unstable solutions. On the other hand, our assumption of a constant spot temperature is supported by the DI results that indicate that hot spots are not required, except during the above-mentioned short period. We plan to reconsider the possible role of hot spots or of a time varying spot temperature in future works by applying the method devised by Messina et al. (2006).

Long-term photometry provides the best data to monitor the variation of the total spotted area in active stars, giving us information on stellar activity cycles. Our modelling indicates a long-term cycle in the K1 IV component of V711 Tau with a period of about $20 \mathrm{yr}$. The present activity cycle begun around 1986 (cf. Figs. 12 and 13) and the Zeeman DI maps obtained since 1990 do not reveal any significant evolution of the largescale photospheric magnetic field pattern up to recent epochs (cf., e.g., Donati 1999; Petit et al. 2004). It will be interesting to look for a possible modification of the global field topology or a polarity switch in the next years when a new cycle is expected to begin.

The absence of a regular latitudinal migration of the largescale toroidal field detected at the surface of the K1 IV component suggests that the dynamo operating in such a star is not solar, i.e., it is not a $\alpha \Omega$ dynamo working in a thin shell at the base of the stellar convection zone or in the overshoot layer. A distributed dynamo, working in the bulk of the stellar convection zone, possibly of the $\alpha^{2} \Omega$ type, seems to be a more plausible candidate (Donati 1999).

It is interesting to correlate the periods of more intense flaring activity, as revealed by radio observations, with the total variation of the spotted area on the K1 IV component. Umana et al. (1995) reported two periods of intense flaring in 1978 and 1989 that correspond to the relative maxima of two short-term oscillations of the total spotted area that took place in 1976-1979 and 1987-1990, respectively. These oscillations may be tracing a short-term cycle with a length of 3-5 years that is superposed on the long-term 20-year cycle (cf. Fig. 13 middle panel). It might be related to the secondary periodicity found in the variation of the ME total spotted area whose significance, as derived from the Scargle-Lomb periodogram, is very low, possibly due to the pronounced variations of the shape and length of the oscillation from one cycle to the next. In any case, short-term cycles with a period of about $5.5 \mathrm{yr}$ in the mean light level and possibly of $3 \mathrm{yr}$ in the polar spot area have been suggested by Henry et al. (1995) and Vogt et al. (1999), respectively.

The spot pattern on the active component of V711 Tau shows a regular longitudinal migration versus time, such as, e.g., in RS CVn (Rodonò et al. 1995), without the sudden switchs of activity between two active longitudes that are reported for other close binary component stars or single rapid rotators (the socalled flip-flop phenomenon; cf., e.g., Berdyugina \& Tuominen 1998; Rodonò et al. 2000; Korhonen et al. 2001).

The variation of the migration rate of the active longitudes has usually been interpreted in terms of surface differential rotation of the active star. Considering the amplitude of the variations reported above, a lower limit for the relative differential rotation is $\Delta \Omega / \Omega \sim 1.2 \times 10^{-3}$, comparable with the amplitude found by Henry et al. (1995). However, this interpretation is questioned by a comparison with the DI maps that reveal no systematic migration in the latitude of the overall spot pattern during the 1982-2001 time interval, but only a latitudinal motion of some of the individual spots on time scales of a few years, i.e., much shorter than the time scale of migration of the active longitudes in our plot. Moreover, no significant variation of the period of the rotational modulation was found, although the frequency resolution was limited as a consequence of the intrinsic evolution of the spot pattern (cf. Sect. 2.2). Therefore, it is probably better to consider the variation of the migration rates of the active longitudes on the K1 IV component of V711 Tau as an intrinsic property of the spot pattern, likely to be related to the slow drift of non-axisymmetric dynamo modes (Küker \& Rüdiger 1999), instead of a measure of the surface differential rotation of the active star. The amplitude and sign of the surface shear can be safely derived only by means of the cross-correlation of successive DI maps, as shown by Petit et al. (2004).

The three-dimensional structure of the active regions in the outer atmosphere of the K1 IV star can be mapped by means of simultaneous UV and EUV observations. Busà et al. (1999) showed that a high-latitude $\left(>67^{\circ}\right)$ chromospheric plage was present in December 1992, possibly superposed onto the polar spot (Donati 1999). Other plages in spatial association with the photospheric active longitudes have been reported by, e.g., Rodonò et al. (1987) and Drake et al. (1994). The studies on the tridimensional structure of the active regions are hampered by the intense flaring activity of the outer atmosphere of the K1 IV star that often makes it impossible to detect the rotational modulation of the UV line fluxes and distorts their profiles (cf., e.g., Dempsey et al. 1996; Wood et al. 1996).

The variation of the orbital period of V711 Tau, as traced by the O-C diagram in the bottom panel of Fig. 13, appears to be correlated with significant changes in the total spotted area and in the configuration of the active longitudes on the surface of the K1 IV star. The change of the slope of the O-C diagram around 1990 indicates a decrease of the orbital period $\left(|\Delta P / P| \simeq 9 \times 10^{-5}\right.$, cf. Frasca \& Lanza 2005) that can be related to the burst of activity that occurred in the 1987-1990 period. It was characterized by a rapid variation of the total spotted area superposed on the gradual rise from the activity minimum in 1986 to the maximum in 1994. This period was also characterized by the detection of hot spots (in the 1988-1990 seasons) and by strong optical (Henry \& Hall 1991), as well as radio flares (Umana et al. 1995). Together with the above results on the spot pattern variations, such observations provide us with substantial evidence for a re-configuration of the surface magnetic fields, possibly following the emergence of a sizeable amount of fresh magnetic flux from the stellar interior. In other words, the observations strongly suggest that the orbital period change is related to a variation of the internal magnetic field configuration of the K1 IV active component, thus supporting some of the theoretical models proposed by Lanza et al. (1998b), Rüdiger et al. (2002), and Lanza \& Rodonò (2004). These models assume that the gravitational quadrupole moment of the active star oscillates versus time inducing a modulation of the orbital period according to the original idea proposed by Matese \& Whitmire (1983). More precisely, when the quadrupole moment is at a maximum, the gravitational pull acting on the companion star is at a maximum, forcing it to move closer and faster around the active star, so the orbital period attains its minimum value. Conversely, when the gravitational quadrupole moment is at a minimum, the acceleration of the companion star is at a minimum and the orbital period is maximum. The variation of the quadrupole moment is produced by a slight change of the figure of equilibrium of the star, due to a variation of the Lorentz force in the stellar convection zone. In these models, the internal magnetic field acts directly on the hydrostatic equilibrium instead of modifying the centrifugal force by transferring some angular momentum back and forth among internal shells of the convection zone, as proposed by Applegate (1992). Indeed, the model proposed by Applegate was shown to be inadequate for RS CVn binary 
systems, and for V711 Tau in particular, by the general analysis of Lanza (2005), who showed that the variation of the internal rotation needed to produce the required quadrupole moment change is two orders of magnitude larger than the amplitude of the observed differential rotation (cf., e.g., Petit et al. 2004) and cannot be supported by the stellar luminosity. These conclusions have recently been confirmed by Lanza (2006) for an arbitrary internal rotation profile, thus generalizing the results of Lanza (2005) that were obtained under the assumption of an angular velocity uniform over cylindrical surfaces co-axial with the stellar rotation axis in the Taylor-Proudman regime.

The duration of the orbital period modulation cycle in V711 Tau is presently unknown, but it is certainly longer than the starspot cycle. Radial velocity monitoring in the forthcoming years will be particularly important to verify the suggestion that the orbital period cycle is about twice the starspot cycle, as observed in the prototype active binary RS CVn and in other systems (cf., e.g., Lanza \& Rodonò 2004, and references therein).

\section{Conclusions}

Our analysis of the long-term optical photometry of the close binary system V711 Tau revealed several interesting aspects of the photospheric spot phenomenology in the very active K1 IV component star. Pooled variance analysis showed that the time scale for the evolution of the spot pattern is of the order of 100200 days. The time baseline to build individual light curves was chosen accordingly, avoiding significant changes of the spot pattern along each light curve. In such a way, 66 seasonal light curves were obtained that almost continuously covered about $26 \mathrm{yr}$ of evolution of the surface activity of the star. The period of the rotational modulation did not show significant variations from season to season, possibly as a consequence of the limited frequency resolution imposed by the quite fast variation of the spot pattern.

The extended data set allowed us a detailed comparison of the longitude distributions of the spotted area derived from photometry with those obtained by means of the DI technique. The results showed that light curve inversion by means of ME and T regularizing techniques can be safely applied to derive the longitude distribution of the spotted area, although with a limited spatial resolution that depends on the complexity of the spot pattern and the spot latitude. In consideration of the systematic effects that limit a precise reconstruction of the spot filling factors by means of the DI techniques, our light curve modelling allowed us a better study of the activity cycle and the overall longitudinal distribution of the active regions on the photosphere of the K1 IV star.

The total spotted area of the K1 IV component showed a modulation with a cyclicity of about 20 years, although only one complete cycle was observed during the extension of our data set. A shorter-term oscillation with a time scale of about 35 years might be present with a shape changing remarkably from one cycle to the next.

Two active longitudes have been detected on the active component, one of which has been dominating from 1976 to 1989. From 1989 on, the two active longitudes have been comparable in their areas up to 2001. Their positions were not fixed with respect to the orbital reference frame, with both longitudes showing a variable migration rate, possibly due to the slow drift of the dominating dynamo modes.

The orbital period decrease that occurred around 1990 was clearly associated with a remarkable re-configuration of the photospheric spot pattern on the K1 IV star characterized by the rise of the area of the second active longitude, a change of their migration rate, a sharp variation of the total spotted area of the star, and intense flaring activity. Together with the results derived by previous studies, this provides compelling evidence for a connection between orbital period modulation and magnetic activity in V711 Tau, and allows us to discriminate among the theoretical models proposed to interpret such a relationship.

Acknowledgements. A.F.L., N.P., S.M., and G.C. wish to dedicate this work to Marcello Rodonò, Professor of Astronomy in the University of Catania and Director of the Astrophysical Observatory of Catania, who suddenly passed away during the preparation of the present paper.

The authors wish to thank the Referee, Dr. Pascal Petit, for his careful reading of the paper and valuable comments.

The acquisition of photometric data over so many years with the Catania APT has been possible thanks to the dedicated and highly competent technical assistance of a number of people, notably P. Bruno, E. Martinetti, and S. Sardone.

Active star research at INAF-Catania Astrophysical Observatory and the Department of Physics and Astronomy of Catania University is funded by MIUR (Ministero della Istruzione, Università e Ricerca), and by Regione Siciliana, whose financial support is gratefully acknowledged.

This research has made use of the ADS-CDS databases, operated at the CDS, Strasbourg, France.

\section{References}

Aarum-Ulvas, V., \& Henry, G. W. 2004, AN, 326, 292

Alekseev I. Yu., \& Kozhevnikova, A. V. 2004, Afz, 47, 519

Andrews, A. D., Rodonò, M., Linsky, J. L., et al. 1988, A\&A, 204, 177

Applegate, J. H. 1992, ApJ, 385, 621

Audard, M., Güdel, M., \& Mewe, R. 2001, A\&A, 365, L318

Bartolini, C., Guarnieri, A., Piccioni, A., et al. 1978, AJ, 83, 1510

Bartolini, C., Blanco, C., Catalano, S., et al. 1983, A\&A, 117, 149

Berdyugina, S. V., \& Tuominen, I. 1998, A\&A, 336, L25

Bopp, B. W., Hall, D. S., Landis, H. J., et al. 1977, AJ, 82, 47

Boyd, L. J., Genet, R. M., Hall, D. S., Busby, M. R., \& Henry, G. W. 1990, IAPPP Comm., 42, 44

Busà, I., Pagano, I., Rodonò, M., Neff, J. E., \& Lanzafame, A. C. 1999, A\&A, 350,571

Chambliss, C. R., \& Detterline, P. J. 1979, IBVS, 3089

Chambliss, C. R., Landis, H. J., Louth, H., et al. 1978, AJ, 83, 1514

Dempsey, C. R., Neff, J. E., Thorpe, M. J., et al. 1996, ApJ, 470, 1172

Diaz-Cordoves, J., Claret, A., \& Gimenez, A. 1995, A\&AS, 110, 329

Dobson, A. K., Donahue, R. A., Radick, R. R., \& Kadlec, K. L. 1990, in The

Sixth Cambridge Symposium on Cool Stars, Stellar Systems and the Sun, ed.

G. Wallerstein, ASP Conf. Ser., 9, 132

Donati, J.-F. 1999, MNRAS, 302, 457

Donati, J.-F., Brown, S. F., \& Semel, S., et al. 1992, A\&A, 265, 682

Donati, J.-F., Collier Cameron, A., Semel, M., et al. 2003, MNRAS, 345, 1145

Donahue, R. A., \& Baliunas, S. L. 1992, ApJ, 393, L63

Donahue, R. A., Dobson, A. K., \& Baliunas, S. L. 1997, Sol. Phys., 171, 191

Dorren, J. D., \& Guinan, E. F. 1982, ApJ, 252, 296

Drake, J. J., Brown, A., Patterer, R. J., et al. 1994, ApJ, 421, L43

Eaton, J. A. 1992, LNP, 397, 15

Eaton, J. A., Henry, G. W., Bell, C., \& Okorogu, A. 1993, AJ, 106, 1181

Fekel, F. C. 1983, ApJ, 268, 274

Flower, P. J. 1996, ApJ, 469, 355

Foing, B. H., Char, S., Ayres, T., et al. 1994, A\&A, 292, 543

Frasca, A., \& Lanza, A. F. 2005, A\&A, 429, 309

García-Alvarez, D., Foing, B. H., Montes, D., et al. 2003, A\&A, 397, 285

Gull, S. F., \& Skilling, J. 1984, in Indirect Imaging, ed. J. A. Roberts (Cambridge: Cambridge Univ. Press), 267

Henry, G. W., \& Hall, D. S. 1991, ApJ, 373, L9

Henry, G. W., Eaton, J. A., Hamer, J., \& Hall, D. S. 1995, ApJS, 97, 513

Horne, J. H., \& Baliunas, S. L. 1986, ApJ, 302, 757

Kopal, Z. 1959, Close Binary Systems (London: Chapman \& Hall Ltd.)

Korhonen, H., Berdyugina, S. V., Strassmeier, K. G., \& Tuominen, I. 2001, A\&A, 379, L30

Küker, M., \& Rüdiger, G. 1999, A\&A, 346, 922

Kurucz, R. 2000, http://cfaku5.harvard.edu

Landis, H. J., Lovell, L. P, Hall, D. S., Henry, G. W., \& Renner, T. R. 1978, AJ, 83,176

Lanza, A. F. 2005, MNRAS, 364, 238

Lanza, A. F. 2006, MNRAS, in press, DOI: 10.1111/j.1365-2966.2006.10415.x

Lanza, A. F., \& Rodonò, M. 2004, AN, 325, 393 
Lanza, A. F., Catalano, S., Cutispoto, G., Pagano, I., \& Rodonò, M. 1998a, A\&A, 332,541

Lanza, A. F., Rodonò, M., \& Rosner, R. 1998b, MNRAS, 296, 893

Lanza, A. F., Rodonò, M., Mazzola, L., \& Messina, S. 2001, A\&A, 376, 1011

Lanza, A. F., Catalano, S., Rodonò, M., et al. 2002, A\&A, 386, 583

Lanzafame, A. C., Busà, I., \& Rodonò, M. 2000, A\&A, 362, 683

Lloyd-Evans, T., \& Koen, M. C. J. 1987, South African Astron. Obs. Circ., 11, 21

Matese, J. J., \& Whitmire, D. P. 1983, A\&A, 117, L7

Mekkaden, M. V., Raveedran, A. V., \& Mohin, S. 1982, J. Astrophys. Astron., 3, 27

Messina, S., Cutispoto, G., Guinan, E. F., Lanza, A. F., \& Rodonò, M. 2006, A\&A, 447, 293

Messina, S., \& Guinan, E. F. 2003, A\&A, 409, 1017

Mohin, S., \& Raveendran, A. V. 1993, A\&AS, 100, 331

Ness, J.-U., Schmitt, J. H. M. M., Burwitz, V., et al. 2002, A\&A, 394, 911

Olsen, E. H. 1983, A\&AS, 54, 55

Osten, R., Brown, A., Ayres, T. R., et al. 2004, ApJS, 153, 317

Parthasarathy, M., Raveedran, A. V., \& Mekkaden, M. V. 1981, A\&AS, 74, 87

Perryman, M. A. C., Lindegren, L., Kovalevsky, J., et al. 1997, A\&A, 323, L49

Petit, P., Donati, J.-F., Wade, G. A., et al. 2004, MNRAS, 348, 1175

Piskunov, N. E., Tuominen, I., \& Vilhu, O. 1990, A\&A, 230, 363
Richards, M., Waltman, E. B., Ghigo, F. D., \& Richards, D. St. P. 2003, ApJS, 147,337

Robinson, R. D., Airapetian, V. S., Maran, S. P., \& Carpenter, K. G. 1996, ApJ, 469,872

Rodonò, M. 1978, unpublished data

Rodonò, M., Cutispoto, G., Pazzani, V., et al. 1986, A\&A, 165, 135

Rodonò, M., Byrne, P. B., Neff, J. E., et al. 1987, A\&A, 176, 267

Rodonò, M., Lanza, A. F., \& Catalano, S. 1995, A\&A, 301, 75

Rodonò, M., Messina, S., Lanza, A. F., Cutispoto, G., \& Teriaca, L. 2000, A\&A, 358,624

Rodonò, M., Cutispoto, G., Lanza, A. F., \& Messina, S. 2001, AN, 322, 333

Rucinski, S. M. 1983, IBVS, 2277

Rüdiger, G., Elstner, D., Lanza, A. F., \& Granzer, Th. 2002, A\&A, 392, 605

Sanz-Forcada, J., Brickhouse, N. S., \& Dupree, A. K. 2002, ApJ, 570, 799

Sarma, M. B. K. 1978, unpublished data

Strassmeier, K. G., \& Bartus, J. 2000, A\&A, 354, 537

Vogt, S. S., Penrod, G. D., \& Hatzes, A. P. 1987, ApJ, 321, 496

Vogt, S. S., Hatzes, A. P., Misch, A. A., \& Kürster, M. 1999, ApJS, 121, 547

Umana, G., Trigilio, C., Tumino, M., Catalano, S., \& Rodonò, M. 1995, A\&A, 298, 143

Wood, B. E., Harper, G. M., Limsky, J. L., \& Dempsey, R. C. 1996, ApJ, 458, 761 
A. F. Lanza et al.: Long-term starspot activity of V711 Tau, Online Material p 1

\section{Online Material}


A. F. Lanza et al.: Long-term starspot activity of V711 Tau, Online Material p 2

Table 1. The light curves of V711 Tau analysed in the present paper.

\begin{tabular}{|c|c|c|c|c|c|c|c|}
\hline $\begin{array}{l}\text { Mean Epoch } \\
\text { (year) }\end{array}$ & $\begin{array}{l}\text { HJD (begin) } \\
(2400000+)\end{array}$ & $\begin{array}{c}\text { HJD (end) } \\
(2400000+)\end{array}$ & $\begin{array}{c}\text { HJD (mean) } \\
(2400000+)\end{array}$ & $M$ & $\begin{array}{c}D_{\mathrm{T}} \\
(\mathrm{cm})\end{array}$ & $P$ & Reference $\left({ }^{a}\right)$ \\
\hline 1975.59 & 42458 & 42801 & 42629 & 24 & $40-90$ & $\mathrm{~S}$ & 1 \\
\hline 1976.13 & 42804 & 42848 & 42826 & 32 & $40-90$ & $\mathrm{~S}$ & 1 \\
\hline 1976.71 & 43008 & 43070 & 43039 & 25 & $25-80$ & $\mathrm{~S}$ & 2 \\
\hline 1976.96 & 43075 & 43184 & 43129 & 23 & $25-80$ & $\mathrm{~S}$ & 2,3 \\
\hline 1977.16 & 43186 & 43217 & 43201 & 20 & $25-80$ & S & 2,3 \\
\hline 1977.70 & 43373 & 43426 & 43399 & 20 & $20-100$ & S-St & 4,8 \\
\hline 1977.90 & 43428 & 43519 & 43473 & 41 & $20-100$ & $\mathrm{~S}-\mathrm{St}$ & $4,5,6,8$ \\
\hline 1978.12 & 43520 & 43589 & 43554 & 33 & $20-100$ & $\mathrm{~S}-\mathrm{St}$ & $4,7,8$ \\
\hline 1978.86 & 43794 & 43855 & 43824 & 30 & $20-90$ & $S$ & 8 \\
\hline 1979.01 & 43856 & 43904 & 43880 & 19 & $20-90$ & $\mathrm{~S}$ & 8 \\
\hline 1979.15 & 43906 & 43952 & 43929 & 21 & $20-90$ & $\mathrm{~S}$ & 8,9 \\
\hline 1979.79 & 44111 & 44219 & 44165 & 60 & $20-90$ & $\mathrm{~S}$ & 8,10 \\
\hline 1980.01 & 44221 & 44268 & 44244 & 50 & $20-90$ & $\mathrm{~S}$ & 8,10 \\
\hline 1980.15 & 44270 & 44322 & 44296 & 64 & $20-90$ & $\mathrm{~S}$ & 8,10 \\
\hline 1980.75 & 44498 & 44529 & 44513 & 17 & $20-90$ & $\mathrm{~S}$ & 8,10 \\
\hline 1980.89 & 44531 & 44599 & 44565 & 44 & $20-90$ & $\mathrm{~S}$ & 8,10 \\
\hline 1981.05 & 44601 & 44645 & 44623 & 25 & $20-90$ & S & 8,10 \\
\hline 1981.18 & 44647 & 44693 & 44670 & 24 & $20-90$ & $S$ & 8,10 \\
\hline 1981.76 & 44866 & 44900 & 44883 & 39 & $20-75$ & $\mathrm{~S}$ & 11 \\
\hline 1981.91 & 44903 & 44970 & 44936 & 27 & $20-75$ & $\mathrm{~S}$ & 11 \\
\hline 1982.04 & 44972 & 44995 & 44983 & 13 & $20-75$ & S-St & $11,12,13$ \\
\hline 1982.14 & 44997 & 45046 & 45021 & 18 & $20-75$ & $S$ & 11,12 \\
\hline 1982.84 & 45253 & 45300 & 45276 & 22 & $10-76$ & $\mathrm{~S}$ & 12,14 \\
\hline 1982.96 & 45301 & 45338 & 45319 & 30 & $10-76$ & $\mathrm{~S}$ & 12,14 \\
\hline 1983.04 & 45340 & 45364 & 45352 & 42 & $10-76$ & $\mathrm{~S}$ & 12,14 \\
\hline 1983.12 & 45366 & 45393 & 45379 & 56 & $10-76$ & $\mathrm{~S}$ & 12,14 \\
\hline 1983.87 & 45629 & 45678 & 45653 & 61 & $20-75$ & $\mathrm{~S}$ & 12,15 \\
\hline 1983.97 & 45680 & 45700 & 45690 & 38 & $20-75$ & $\mathrm{~S}$ & 12,15 \\
\hline 1984.09 & 45702 & 45765 & 45733 & 44 & $20-75$ & $\mathrm{~S}$ & 12,15 \\
\hline 1984.81 & 45971 & 46020 & 45995 & 41 & $20-75$ & $\mathrm{~S}$ & 12,15 \\
\hline 1985.01 & 46021 & 46122 & 46071 & 43 & $20-75$ & S & 12,15 \\
\hline 1985.72 & 46286 & 46370 & 46328 & 57 & $20-75$ & S & 12,15 \\
\hline 1987.10 & 46800 & 46866 & 46833 & 45 & $20-75$ & $\mathrm{~S}$ & 12,15 \\
\hline 1987.84 & 47072 & 47137 & 47104 & 35 & $20-75$ & $\mathrm{~S}$ & $12,15,16$ \\
\hline 1988.07 & 47139 & 47233 & 47186 & 56 & $20-75$ & $\mathrm{~S}$ & $12,15,16$ \\
\hline 1988.77 & 47415 & 47474 & 47444 & 63 & $34-40$ & $S$ & 12,16 \\
\hline 1988.93 & 47476 & 47528 & 47502 & 38 & $34-40$ & S & 12,16 \\
\hline 1989.10 & 47530 & 47599 & 47564 & 45 & $34-40$ & $\mathrm{~S}$ & 12,16 \\
\hline 1989.77 & 47778 & 47839 & 47808 & 60 & $34-40$ & $\mathrm{~S}$ & 12,16 \\
\hline 1989.94 & 47840 & 47899 & 47869 & 65 & $34-40$ & $\mathrm{~S}$ & 12,16 \\
\hline 1990.10 & 47901 & 47958 & 47929 & 41 & $34-40$ & $\mathrm{~S}$ & 12,16 \\
\hline 1990.79 & 48167 & 48198 & 48182 & 32 & $25-40$ & $\mathrm{~S}$ & $12,16,17$ \\
\hline 1990.94 & 48199 & 48270 & 48234 & 46 & $25-40$ & $\mathrm{~S}$ & $12,16,17$ \\
\hline 1991.12 & 48271 & 48335 & 48303 & 65 & $25-40$ & $\mathrm{~S}$ & $12,16,17$ \\
\hline 1991.89 & 48531 & 48638 & 48584 & 35 & $25-80$ & $\mathrm{~S}$ & 16,17 \\
\hline 1992.10 & 48640 & 48681 & 48660 & 16 & $25-80$ & $\mathrm{~S}$ & 16,17 \\
\hline 1992.78 & 48874 & 48939 & 48906 & 38 & $25-80$ & S & 16,17 \\
\hline 1992.92 & 48941 & 48980 & 48960 & 20 & $25-80$ & $\mathrm{~S}$ & 16,17 \\
\hline 1993.10 & 48981 & 49066 & 49023 & 18 & $25-80$ & $\mathrm{~S}$ & 16,17 \\
\hline 1993.79 & 49260 & 49295 & 49277 & 24 & $25-80$ & $\mathrm{~S}$ & 17 \\
\hline 1993.90 & 49296 & 49340 & 49318 & 24 & $25-80$ & S & 17 \\
\hline 1994.07 & 49341 & 49417 & 49379 & 30 & $25-80$ & S & 17 \\
\hline 1995.76 & 49980 & 50011 & 49995 & 27 & $25-80$ & $\mathrm{~S}$ & 17 \\
\hline 1996.09 & 50100 & 50131 & 50115 & 14 & $25-80$ & S & 17 \\
\hline 1996.90 & 50390 & 50435 & 50412 & 45 & $25-80$ & $\mathrm{~S}$ & 17 \\
\hline 1997.05 & 50437 & 50501 & 50469 & 45 & $25-80$ & $\mathrm{~S}$ & 17 \\
\hline 1997.81 & 50726 & 50765 & 50745 & 35 & $25-80$ & S & 17 \\
\hline 1997.95 & 50766 & 50829 & 50797 & 46 & $25-80$ & $\mathrm{~S}$ & 17 \\
\hline 1998.13 & 50830 & 50892 & 50861 & 11 & $25-80$ & $\mathrm{~S}$ & 17 \\
\hline 1998.84 & 51061 & 51180 & 51120 & 66 & $25-80$ & $\mathrm{~S}$ & 17 \\
\hline 1999.08 & 51181 & 51235 & 51208 & 28 & $25-80$ & $S$ & 17 \\
\hline 1999.85 & 51458 & 51520 & 51489 & 31 & $25-80$ & $\mathrm{~S}$ & 17 \\
\hline 2000.07 & 51521 & 51621 & 51571 & 29 & $25-80$ & $\mathrm{~S}$ & 17 \\
\hline 2000.81 & 51804 & 51881 & 51842 & 38 & $25-80$ & $\mathrm{~S}$ & 17 \\
\hline 2001.00 & 51881 & 51942 & 51911 & 32 & $25-80$ & $\mathrm{~S}$ & 17 \\
\hline
\end{tabular}


A. F. Lanza et al.: Long-term starspot activity of V711 Tau, Online Material $p 3$

Table 1. continued.

\begin{tabular}{cccccccc}
\hline \hline $\begin{array}{c}\text { Mean Epoch } \\
\text { (year) }\end{array}$ & $\begin{array}{c}\text { HJD (begin) } \\
(2400000+)\end{array}$ & $\begin{array}{c}\text { HJD (end) } \\
(2400000+)\end{array}$ & $\begin{array}{c}\text { HJD (mean) } \\
(2400000+)\end{array}$ & $M$ & $\begin{array}{c}D_{\mathrm{T}} \\
(\mathrm{cm})\end{array}$ & $P$ & Reference $\left(^{a}\right)$ \\
\hline 2001.80 & 52160 & 52247 & 52203 & 31 & $25-80$ & $\mathrm{~S}$ & 17 \\
\hline
\end{tabular}

${ }^{a}$ References: 1. Bopp et al. (1977); 2. Landis et al. (1978); 3. Parthasarathy et al. (1981); 4. Bartolini et al. (1978); 5. Rodonò (1978); 6. Sarma (1978); 7. Chambliss et al. (1978); 8. Bartolini et al. (1983); 9. Chambliss \& Detterline (1979); 10. Mekkaden et al. (1982); 11. Rodonò et al. (1986); 12. Mohin \& Raveendran (1993); 13. Rucinski (1983); 14. Andrews et al. (1988); 15. Boyd et al. (1990); 16. Henry et al. (1995); 17. present paper.

Table 3. The $\chi^{2}$ of the light curve best fits with the ME and T regularizing criteria, respectively.

\begin{tabular}{cccccc}
\hline \hline Light curve & $\chi_{\mathrm{ME}}^{2}$ & $\chi_{\mathrm{T}}^{2}$ & Light curve & $\chi_{\mathrm{ME}}^{2}$ & $\chi_{\mathrm{T}}^{2}$ \\
\hline 1975.59 & 0.841 & 0.818 & 1987.84 & 3.578 & 3.471 \\
1976.13 & 0.641 & 0.605 & 1988.07 & 1.013 & 0.967 \\
1976.71 & 3.059 & 2.879 & 1988.77 & 0.878 & 0.780 \\
1976.96 & 1.945 & 1.822 & 1988.93 & 2.644 & 2.529 \\
1977.16 & 0.808 & 0.717 & 1989.10 & 3.042 & 2.817 \\
1977.70 & 1.285 & 1.235 & 1989.77 & 1.071 & 0.965 \\
1977.90 & 1.227 & 1.150 & 1989.94 & 2.197 & 2.032 \\
1978.12 & 0.764 & 0.707 & 1990.10 & 3.337 & 3.275 \\
1978.86 & 0.654 & 0.578 & 1990.79 & 0.370 & 0.237 \\
1979.01 & 0.498 & 0.390 & 1990.94 & 0.890 & 0.830 \\
1979.15 & 1.110 & 0.987 & 1991.12 & 1.518 & 1.463 \\
1979.79 & 1.177 & 1.066 & 1991.89 & 0.470 & 0.464 \\
1980.01 & 2.908 & 2.700 & 1992.10 & 0.392 & 0.338 \\
1980.15 & 2.382 & 2.175 & 1992.78 & 0.673 & 0.671 \\
1980.75 & 0.174 & 0.082 & 1992.92 & 0.575 & 0.577 \\
1980.89 & 0.727 & 0.640 & 1993.10 & 0.950 & 0.938 \\
1981.05 & 1.280 & 1.233 & 1993.79 & 0.209 & 0.180 \\
1981.18 & 1.695 & 1.595 & 1993.90 & 0.970 & 0.948 \\
1981.76 & 0.800 & 0.702 & 1994.07 & 0.311 & 0.313 \\
1981.91 & 0.720 & 0.693 & 1995.76 & 0.315 & 0.345 \\
1982.04 & 1.988 & 1.809 & 1996.09 & 0.429 & 0.485 \\
1982.14 & 2.260 & 2.010 & 1996.90 & 0.680 & 0.747 \\
1982.84 & 0.982 & 0.858 & 1997.05 & 0.558 & 0.719 \\
1982.96 & 0.984 & 0.843 & 1997.81 & 0.445 & 0.611 \\
1983.04 & 0.678 & 0.621 & 1997.95 & 0.383 & 0.488 \\
1983.12 & 0.853 & 0.808 & 1998.13 & 0.267 & 0.227 \\
1983.87 & 0.459 & 0.412 & 1998.84 & 0.598 & 0.764 \\
1983.97 & 1.986 & 1.888 & 1999.08 & 0.677 & 0.848 \\
1984.09 & 1.031 & 0.990 & 1999.85 & 0.519 & 0.541 \\
1984.81 & 0.774 & 0.664 & 2000.07 & 0.832 & 0.772 \\
1985.01 & 3.945 & 3.706 & 2000.81 & 0.818 & 1.018 \\
1985.72 & 1.264 & 1.191 & 2001.00 & 0.900 & 1.051 \\
1987.10 & 1.426 & 1.275 & 2001.80 & 0.407 & 0.374 \\
\hline & & & & &
\end{tabular}


A. F. Lanza et al.: Long-term starspot activity of V711 Tau, Online Material p 4

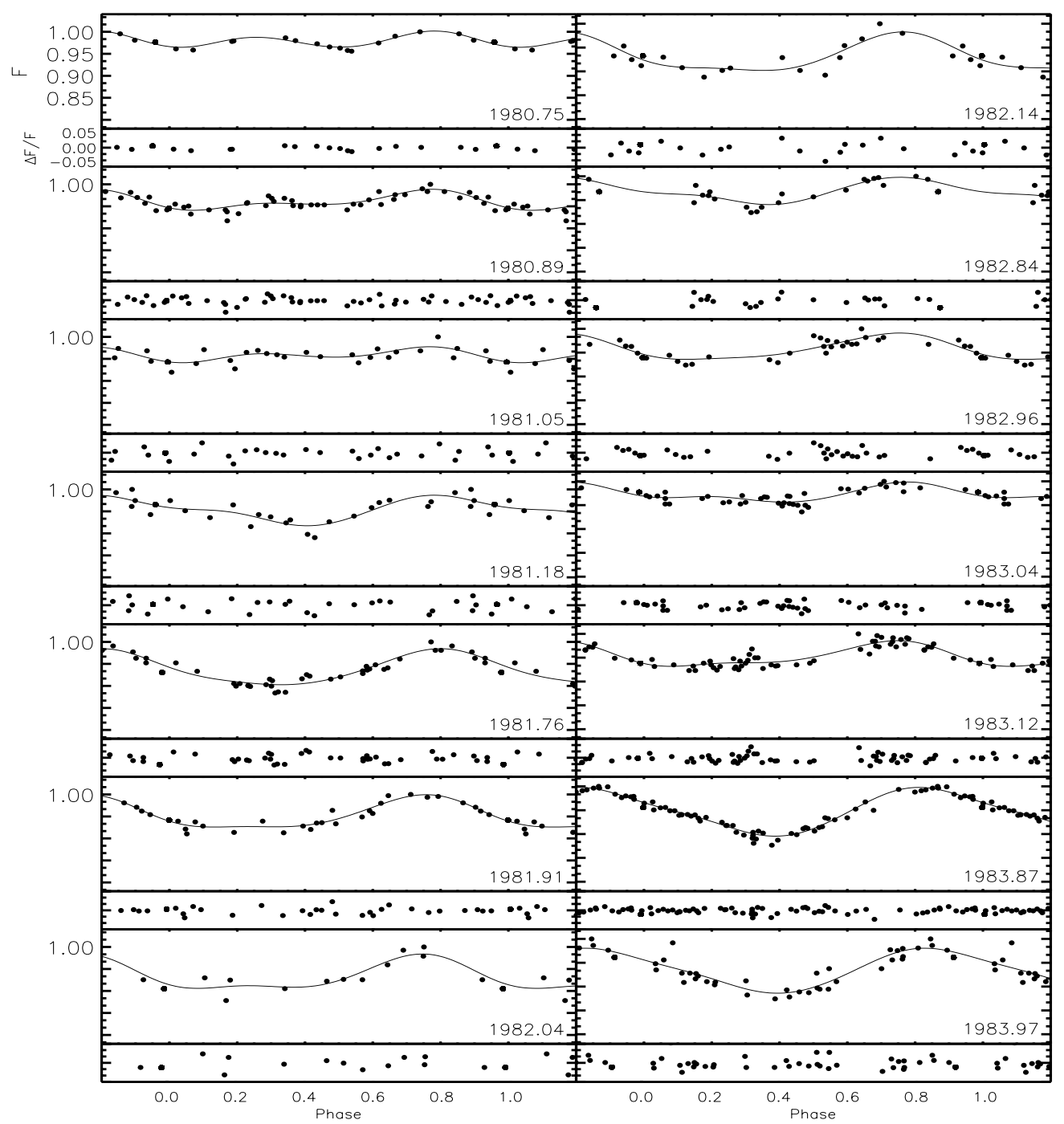

Fig. 4. The same as Fig. 3 for the light curves from the second part of the 1980 season to the 1983 season. 
A. F. Lanza et al.: Long-term starspot activity of V711 Tau, Online Material p 5

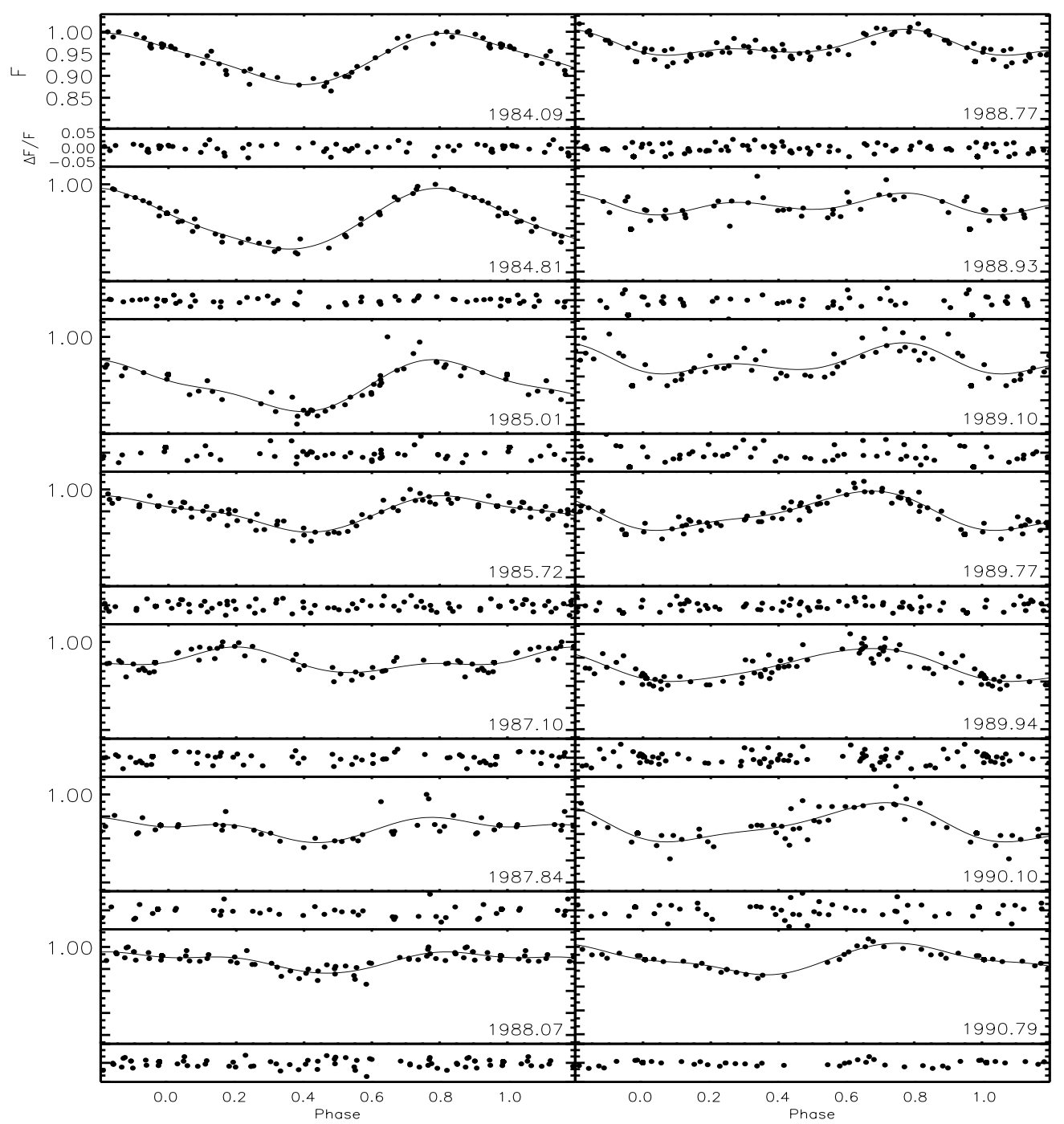

Fig. 5. The same as Fig. 3 for the light curves from 1984 to the first part of the 1990 season. 
A. F. Lanza et al.: Long-term starspot activity of V711 Tau, Online Material p 6

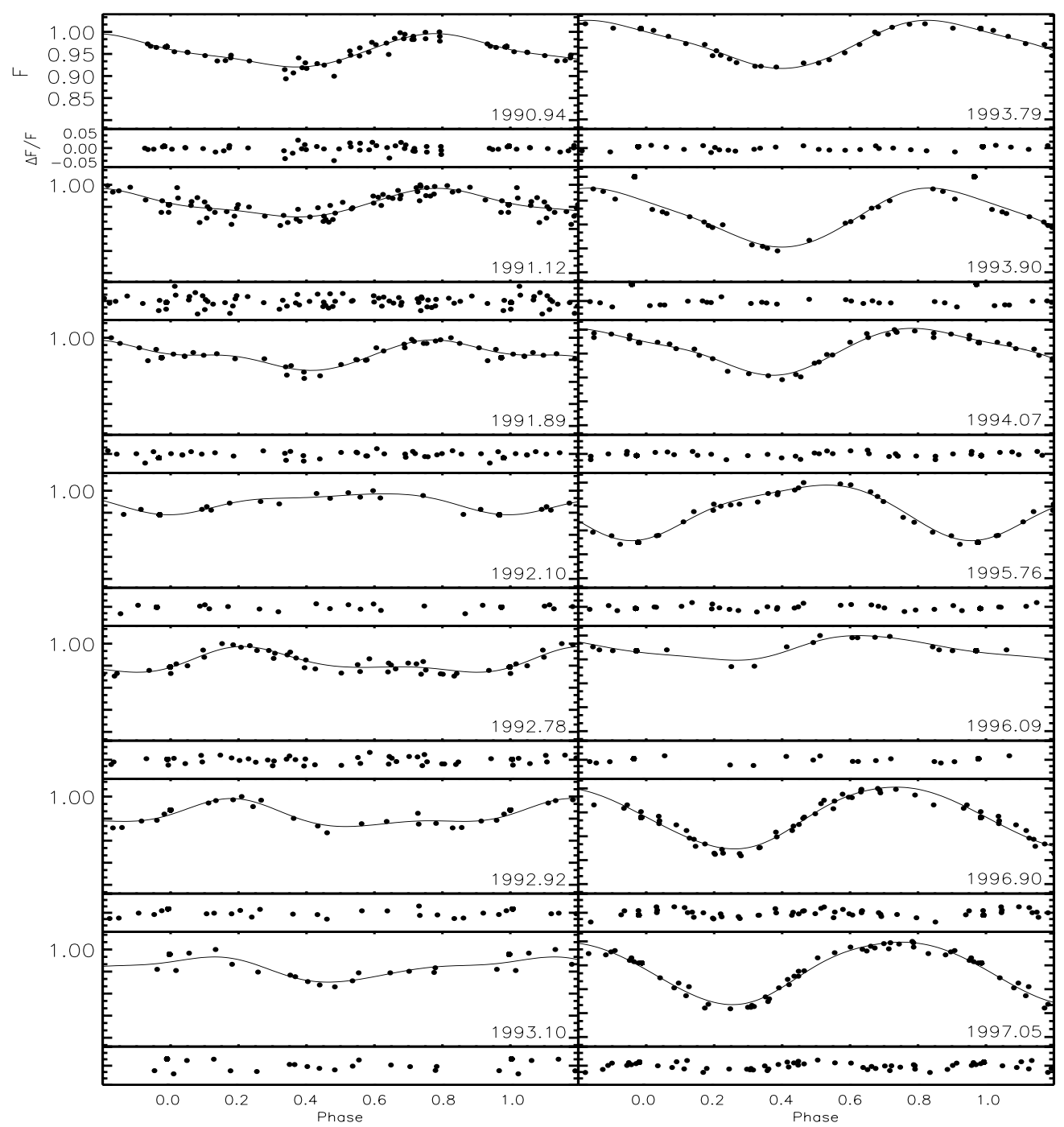

Fig. 6. The same as Fig. 3 for the light curves from the final part of the 1990 season to the beginning of the 1997 season. 
A. F. Lanza et al.: Long-term starspot activity of V711 Tau, Online Material $p 7$

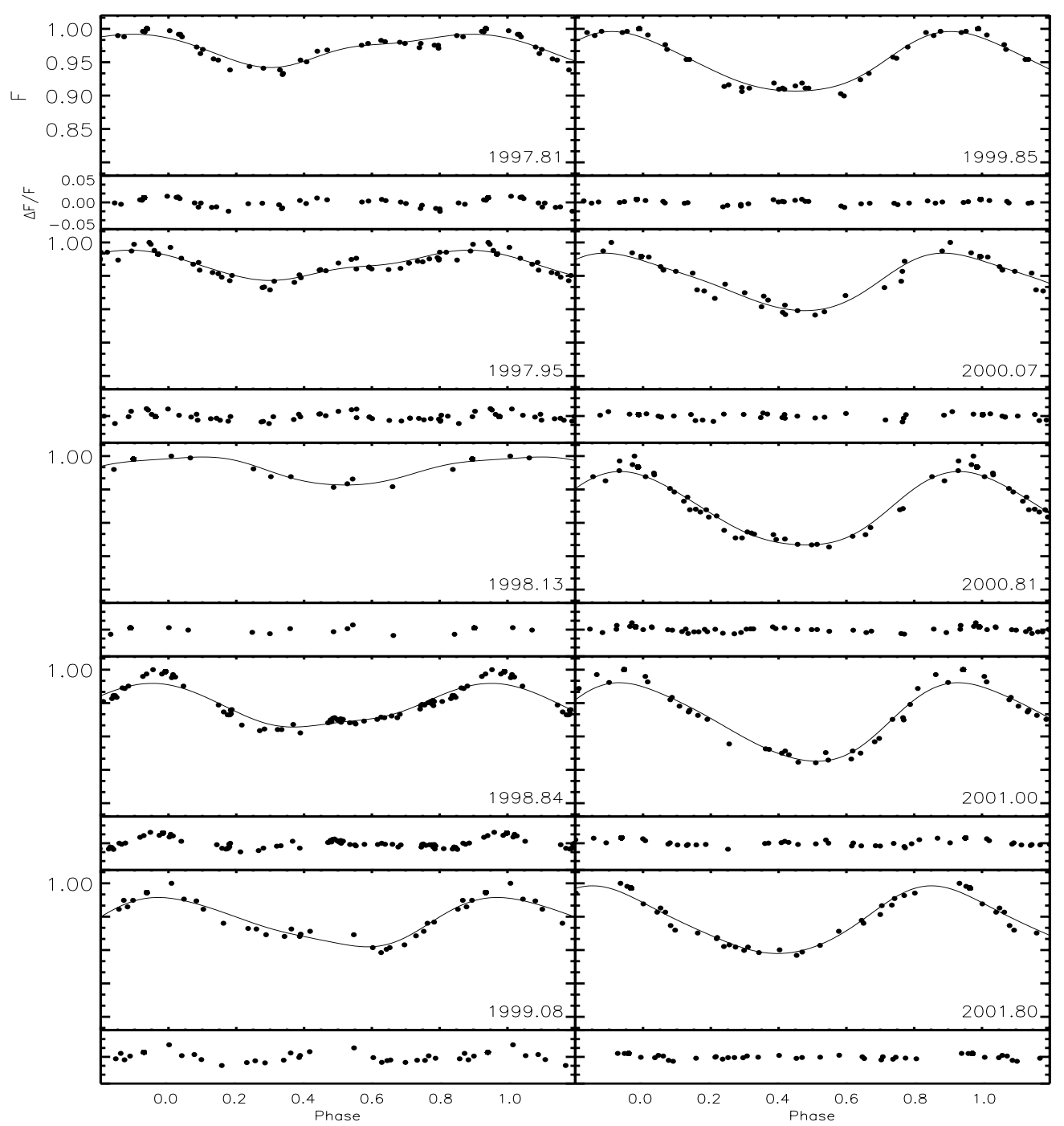

Fig. 7. The same as Fig. 3 for the light curves from the second part of the 1997 season to the 2001 season. 
A. F. Lanza et al.: Long-term starspot activity of V711 Tau, Online Material p 8

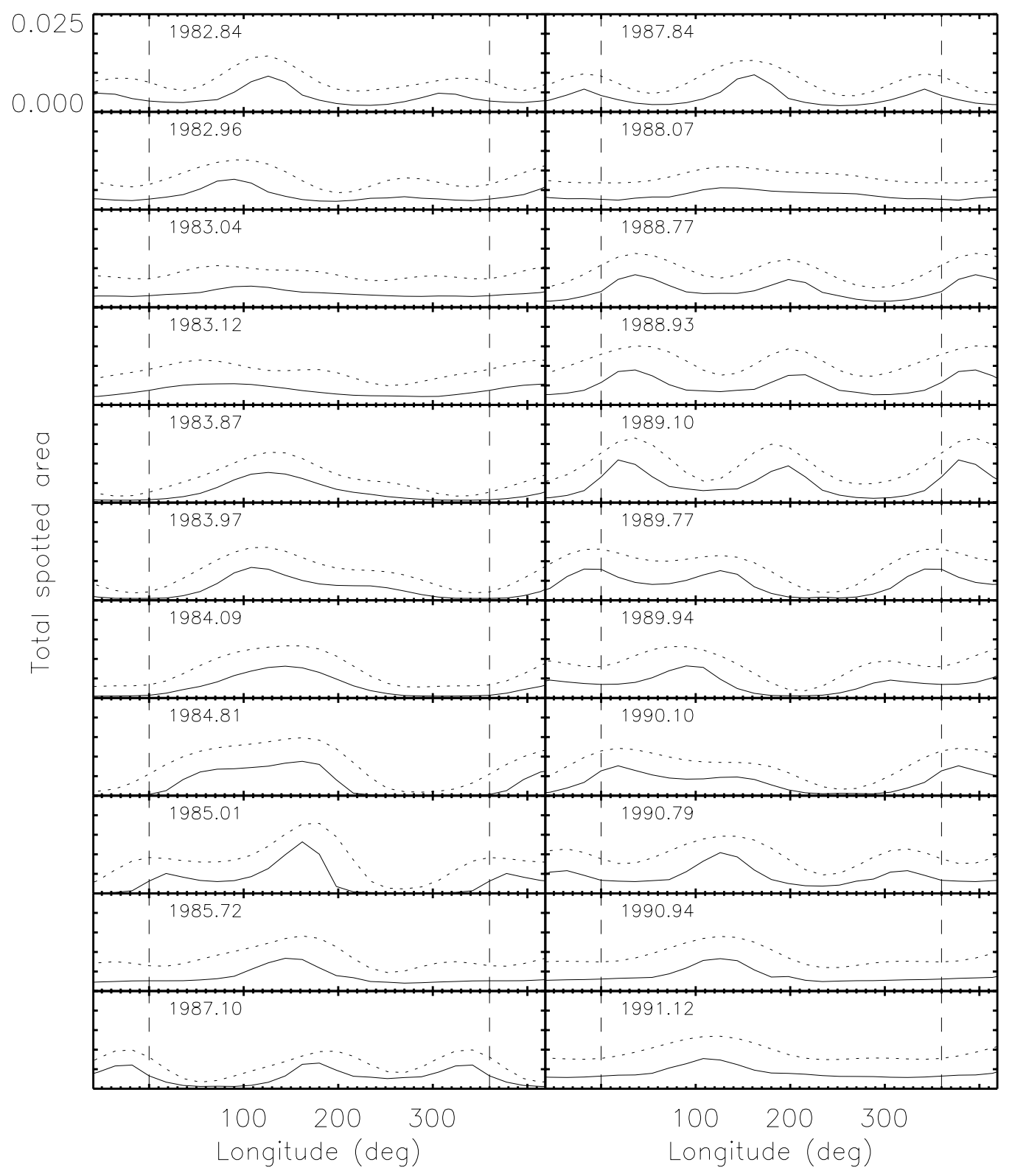

Fig. 10. The same as Fig. 9 for the light curve models from 1982.84 to 1991.12. 
A. F. Lanza et al.: Long-term starspot activity of V711 Tau, Online Material $p 9$

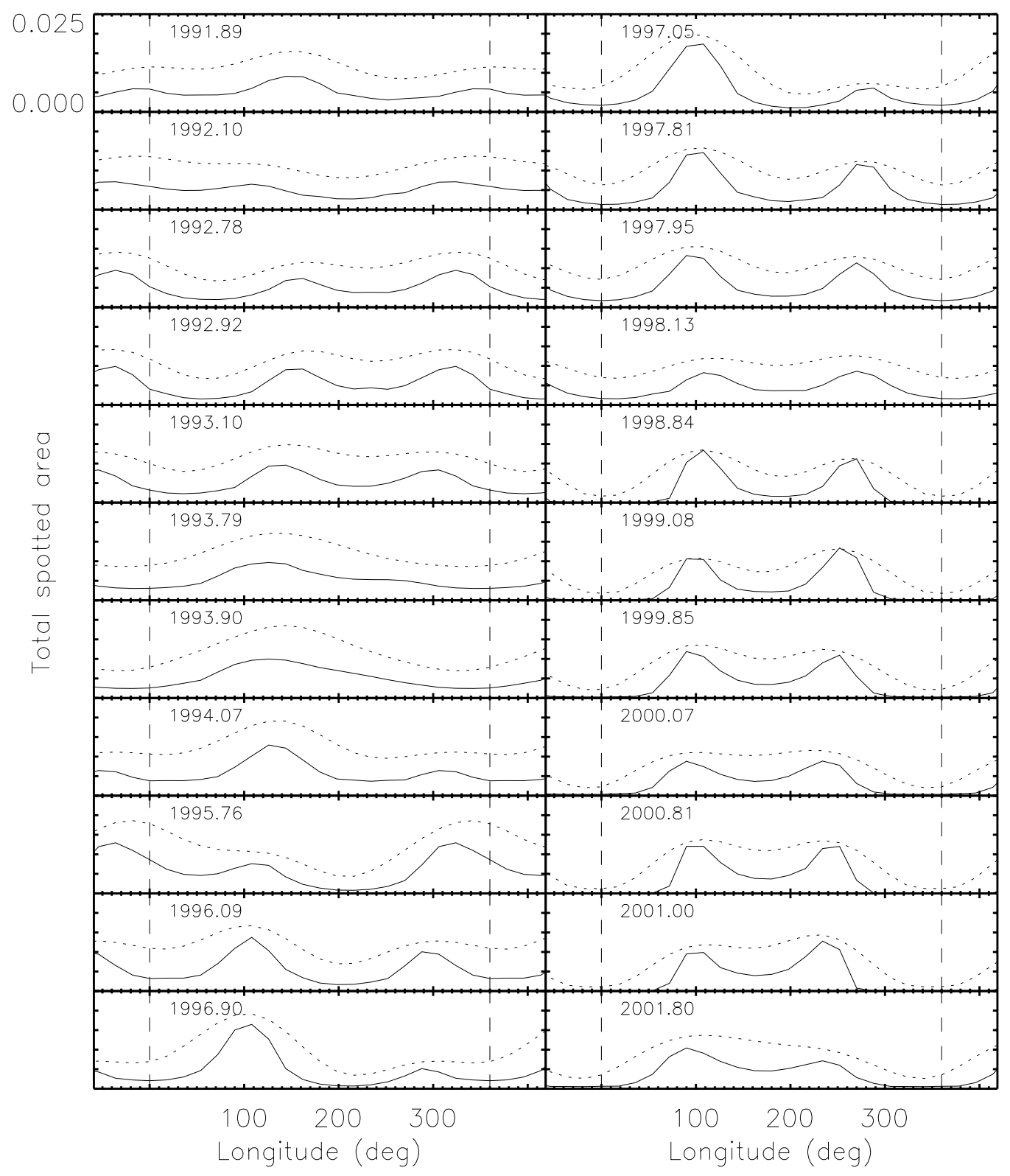

Fig. 11. The same as Fig. 9 for the light curve models from 1991.89 to 2001.80. 